\title{
INTEGRAÇÃO IOT E GIS: UMA REVISÃO SISTEMÁTICA DE SUAS APLICAÇÕES
}

\author{
Iran Carlos Caria Sacramento \\ Universidade Federal da Bahia, Programa de Pós Graduação em Engenharia Civil (PPEC) \\ iransacramento@hotmail.com \\ Vivian de Oliveira Fernandes \\ Universidade Federal da Bahia, Departamento de Engenharia de Transportes e Geodésia \\ vivian.fernandes@ufba.br \\ Emerson de Andrade Marques Ferreira \\ Universidade Federal da Bahia, Departamento de Construção e Estruturas \\ ferreiraeam@gmail.com
}

\begin{abstract}
RESUMO
A Internet of Things (loT) está levando inúmeros benefícios para a vida humana por meio de serviços inteligentes. Com o crescimento massivo dos grandes conjuntos de dados dinâmicos, a aplicação de novos métodos para integração de tecnologias vem se tornando mais diversificados do que antes. Assim, o Geographic Information System (GIS) aparece como uma plataforma acessível, visto que, os métodos de gerenciamento e/ou monitoramento requerem uma múltipla fusão de informações. Este artigo visa analisar através da estatística descritiva as diferentes abordagens, contribuições e soluções quanto as aplicações relacionadas à integração da loT e o GIS. A Revisão Sistemática de Literatura (RSL) foi utilizada enquanto método de pesquisa, envolvendo as seguintes etapas: (a) planejamento da revisão, (b) técnica de seleção e (c) análise dos resultados. Os resultados possibilitaram evidenciar lacunas de pesquisa, vantagens e desvantagens quanto aos métodos investigados. As áreas de aplicação identificadas envolvem a segurança, operação e manutenção, saúde, tráfego e mobilidade urbana, arquitetura para implementação de sistema, agricultura de precisão e monitoramento ambiental. Considerase que as abordagens relativas a loT e ao GIS dispõe de uma grande diversidade de ferramentas para a exploração destas tecnologias, demonstrando que esta integração está em pleno desenvolvimento.
\end{abstract}

Palavras-chave: IoT. GIS. Revisão Sistemática da Literatura (RSL).

\section{IOT AND GIS INTEGRATION: A SYSTEMATIC REVIEW OF ITS APPLICATIONS}

\begin{abstract}
The Internet of Things (loT) is bringing numerous benefits to human life through intelligent services. With the massive growth of large dynamic data sets, the application of new methods for integrating technologies has become more diverse than before. Thus, the Geographic Information System (GIS) appears as an accessible platform, since the methods of management and / or monitoring require a multiple fusion of information. This article aims to analyze through descriptive statistics the different approaches, contributions and solutions regarding the applications related to the integration of IOT and GIS. The Systematic Literature Review (RSL) was used as a research method, involving the following steps: (a) review planning, (b) selection technique and (c) analysis of the results. The results made it possible to highlight research gaps, advantages and disadvantages regarding the investigated methods. The application areas identified involve safety, operation and maintenance, health, traffic and urban mobility, architecture for system implementation, precision agriculture and environmental monitoring. IoT and GIS approaches are considered to have a wide range of tools for the exploitation of these technologies, demonstrating that this integration is in full development.
\end{abstract}

Keywords: IoT. GIS. Systematic Literature Review (RSL).

\section{INTRODUÇÃO}

As transformações nas cidades ocorrem em consequência das interações promovidas pela relação homem-natureza. Tais intervenções, organizam, desorganizam, significam, ou ressignificam um ambiente construído, levando pesquisadores a repensarem novos modelos de organização, entender novos hábitos, padrões, comportamentos e tendência das sociedades (SILVA, 2011). De acordo com Dubois et al. (2004), a vulnerabilidade urbana das cidades é uma noção complexa, na medida em que as vulnerabilidades se encontram territorializadas, cada local irá possuir suas próprias características,

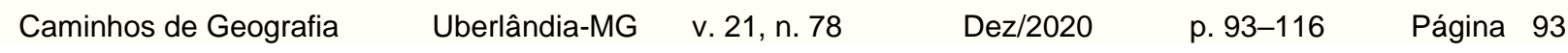


as quais, vão determinar seus limites e guiar as respostas de prevenção em face a sua diversidade de problemas.

Os relatórios mais recentes apontam que até 2050 a população mundial crescerá cerca de $33 \%$, aumentando de 7,3 bilhões para 9,8 bilhões de pessoas, superando de imediato os índices divulgados em 2008 pela Organização das Nações Unidas (ONU, 2008). Diante das perspectivas, a ONU (2017) ainda considera que os principais centros urbanos do mundo se tornarão em megacidades. Em 2016 já existiam $31 \mathrm{com}$ mais de 10 milhões de habitantes, que aumentam para 41 até 2030 (ONU, 2017). Para Suakanto et al (2013), esta transição trouxe consigo um novo panorama de classificação das cidades, ou seja, as cidades altamente urbanizadas, onde as demandas de infraestrutura para os cidadãos aumentarão significativamente, e em algumas delas, seus sistemas, instalações e recursos estão envelhecidos, necessitando de ampliações e modernizações (AMORIM, 2016).

Nos últimos anos a Internet of Things (IOT) apresentou contribuições nos aspectos relacionados a sociedade, como por exemplo: em cidades, automações residenciais, monitoramento ambiental, universidades, ambientes agrícolas, construções inteligentes, transporte inteligente, operação e manutenção, logística, segurança e centros de saúde (YUAN et al., 2013; GUBBI et al., 2013). Apesar das diferentes motivações, os seus domínios de aplicação possuem um objetivo em comum: prover serviços inteligentes para aumentar a qualidade da vida humana (BELLO et al., 2017; FORTINO et al., 2017).

Entretanto, com o crescimento massivo dos grandes conjuntos de dados dinâmicos, rápido desenvolvimento das redes de sensores e a possibilidade da transmissão de dados em tempo real, os métodos de aquisição de dados tornam-se mais diversificados do que antes (PASQUALE et al., 2008; GUNDUZ et al., 2017). Neste sentido, o Geographic Information System (GIS) aparece como uma plataforma acessível para muitas áreas de conhecimento, pois pode usufruir destes dados, tendo em vista que os métodos de gerenciamento e/ou monitoramento das cidades requerem uma múltipla fusão de informações, adquiridas por meio de dados cartográficos, modelos geométricos ou tridimensionais, objetos virtuais ou objetos reais (YUAN et al., 2012; GUNDUZ et al., 2017; ISIKDAG, 2015).

Assim, torna-se relevante compreender os paradigmas que abarcam os diferentes métodos, tecnologias e aplicações integradas nos domínios loT e GIS. O cenário aqui descrito torna-se atrativo, visto existência na camada loT de um ecossistema que contém objetos inteligentes equipados com sensores, redes e tecnologias de processamento, enquanto o GIS aparece como uma ferramenta de grande potencial técnico, um fenômeno mundial amplamente utilizado que contribui em várias áreas de atuação e desenvolve novos insights de pesquisa (BURROUGH e MCDONNELL, 1998).

Deste modo, este artigo busca analisar através da estatística descritiva as diferentes abordagens, contribuições e soluções quanto as aplicações relacionadas à integração da loT e o GIS. Entende-se, que a Revisão Sistemática da Literatura (RSL), possibilitará diagnosticar tendências operacionais e principais lacunas de pesquisa quanto as aplicações estudadas. O presente estudo insere-se em uma pesquisa de doutorado, desenvolvida no Programa de Pós-Graduação em Engenharia Civil (PPEC) da Universidade Federal da Bahia (UFBA).

\section{METODO DE SELEÇÃO DA PESQUISA}

Segundo Kitchenham (2004), a Revisão Sistemática da Literatura (RSL) é um importante instrumento para identificar, avaliar e interpretar questões de pesquisa, seja ela temáticas específicas ou fenômenos de interesse, avaliando assim, o que já existe de disponível e relevante, bem como a descoberta de novas lacunas de pesquisa.

Os critérios adotados na revisão envolvem procedimentos: (i) planejamento da revisão; (ii) condução da revisão; e (iii) análise dos resultados. Tais procedimentos buscam entender as seguintes questões: a) Quais seriam as tecnologias para obtenção de dados, softwares, sensores, metodologias de armazenamento, plataformas de visualização, transmissão de dados, formato e interface em loT? b) Como integrar dados de sensores loT em tempo real a um banco de dados espacial? c) Quais os tipos de sensores e plataformas que poderiam atender a demanda supracitada? d) Quais seriam as plataformas e softwares que permitem o desenvolvimento de uma nova arquitetura de sistemas a baixo custo?

\section{Planejamento da revisão}

O planejamento de busca foi estruturado para identificar as múltiplas fontes de evidência, visando discriminar: (i) objeto da pesquisa; (ii) estratégias de busca; (iii) listagem das bases pesquisadas (iv) critérios para inclusão e exclusão; (v) sumarização dos resultados.

\begin{tabular}{|c|c|}
\hline Caminhos de Geografia & Uberlândia-MG \\
\hline
\end{tabular}


Definiu-se o objetivo da RSL em analisar através da estatística descritiva as diferentes abordagens, contribuições e soluções quanto as aplicações relacionadas à integração da loT e o GIS. Enquanto estratégia de busca definiu-se que os trabalhos deveriam estar publicados em base de periódicos científicos e contemplar o intervalo temporal de 2000 a 2018. Tal escolha é condicionada em consequência da expressão Internet of Things (IoT) ter sido utilizada pela primeira vez no Auto-ID Center do Massachusetts Institute of Technology (MIT), em 1999, e assim chamar a atenção de pesquisadores e profissionais da área de tecnologia (ASHTON, 2009; YUAN et al., 2012), a ampla disseminação na metade do ano 2000 quanto a aplicação das Tecnologias da Informação e Comunicação (TIC) para o gerenciamento urbano por meio de combinações inteligentes (GIFFINGER et al., 2007) e as percepções enfáticas quanto a potencialidade do Geographic Information System (GIS) trazidas na segunda edição do livro Principles of Geographical Information Systems por Burrough e McDonnel em 1998.

Mediante isso, os termos de busca empregados foram: "Internet of Things", "IoT", "Geographic Information System", "GIS", "WebGIS", "Sensor", "Real-time Data", "Building Information Modeling", "BIM". As buscas foram efetuadas nas bases de dados cientificas Scopus, Science Direct e Web of Science. A utilização das bases é justificada em virtude da facilidade de acesso e filtragem dos artigos, bem como a viabilidade de suporte na seleção de estudos relevantes. Alguns critérios foram definidos para a exclusão dos trabalhos selecionados a saber: (i) estudos de revisão de literatura (ii) repetição de trabalhos entre bases; (iii) indisponibilidade para download completo dos trabalhos. Enquanto critérios de inclusão, definiu-se: (i) trabalhos que retratem uma aplicação associada a integração da loT com o GIS; (ii) trabalhos que possuam o termo de busca ao menos no título, resumo ou palavras-chave; (iii) trabalhos online publicados entre os anos 2000 e 2018. A Figura 1 apresenta de modo esquemático o desenvolvimento das etapas adotadas.

Figura 1 - Critérios utilizados para seleção das pesquisas.

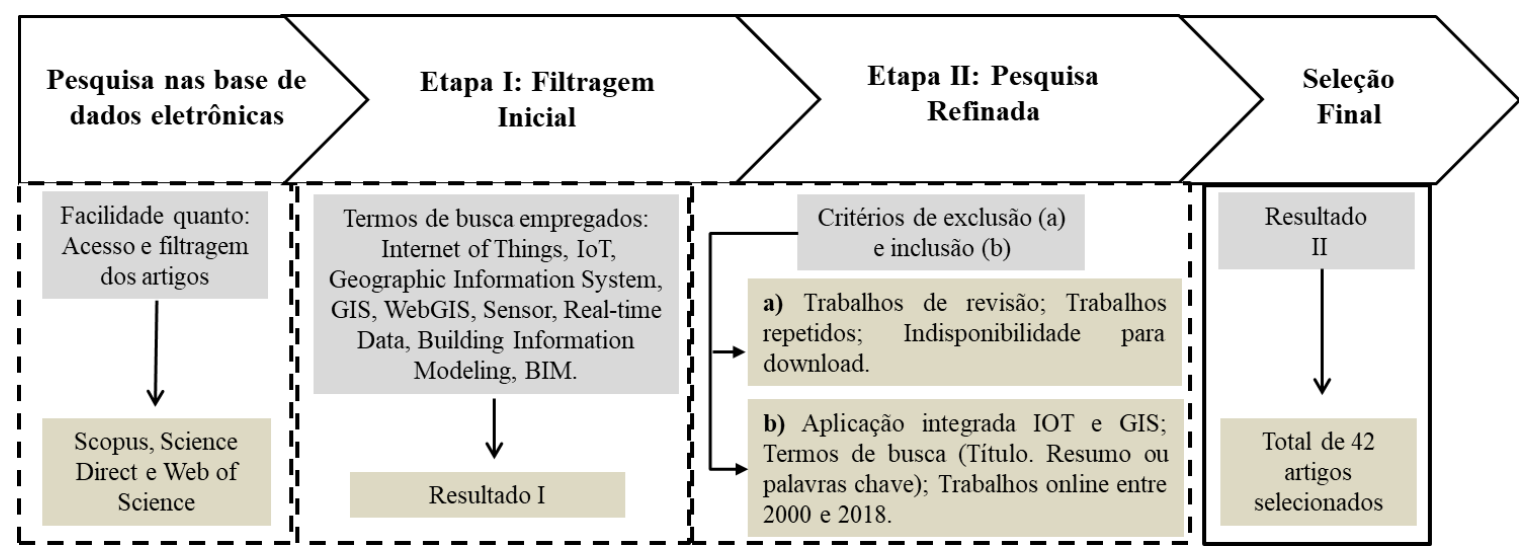

Fonte - Os autores.

\section{Técnica de seleção da pesquisa}

Para obter as literaturas integradas utilizou-se as seguintes combinações: Internet of things e/ou loT "AND" Geographic Information System e/ou GIS "AND" Building Information Modeling e/ou BIM. Em seguida utilizou-se os termos Geographic Information System e/ou GIS "AND" Real Time "AND" Internet of things e/ou loT e por fim, WebGIS "AND" Real Time "AND" Sensor. Inicialmente foram encontradas 414 publicações, todas associadas a artigos completos ou resumos expandidos.

A partir da aplicação dos critérios de exclusão (i, ii e iii) detectou-se 34 pesquisas voltadas a revisão de literatura, 66 pesquisas com repetições nas bases e o número de 108 trabalhos indisponíveis para download. Perfazendo assim, um total de 206 artigos para análise (Figura 2).

Em seguida com a aplicação dos critérios de inclusão a amostra resultante abarcou um total de 42 publicações, sendo 20 para resumos expandidos e 24 para artigos completos, os quais, agruparam as áreas de segurança, operação e manutenção, saúde, tráfego e mobilidade urbana, arquitetura para implementação de sistema, agricultura de precisão e monitoramento ambiental.

1 Justifica-se a inclusão do Building Information Modeling (BIM), pois de acordo com Isikdag (2015) o BIM aborda em suas discussões problemas relacionados a compartilhamento de informações em tempo real e quanto a interoperabilidade de dados. 
Figura 2 - Fluxo da seleção das pesquisas.
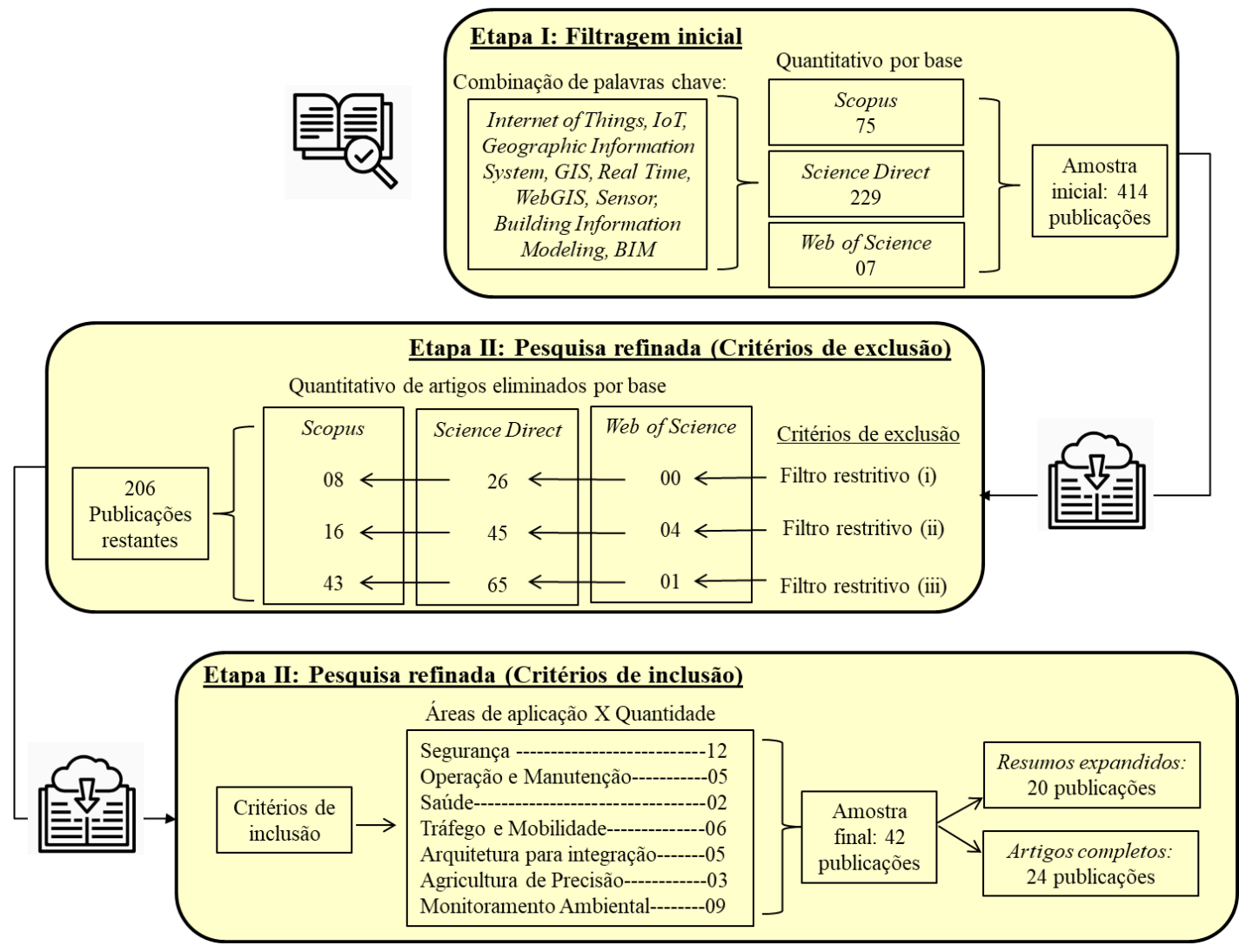

Fonte - Os autores.

Não é de surpreender a vasta quantidade de pesquisas relacionadas a aplicações em Internet of Things (IoT) e Geographic Information System (GIS) em diferentes campos, principalmente, quando se trata do crescimento exponencial para o uso de Big Data e dos dados coletados por sensores em todo mundo (AHAMED, 2017). A figura 3 apresenta a distribuição relacionada as publicações destinadas a integração no âmbito da loT e GIS. Para o quantitativo apresentado, destaca-se a China com um total de 17 publicações, seguida pela Itália com 7 e Turquia com 3 publicações.

Figura 3 - Distribuição das publicações por países.

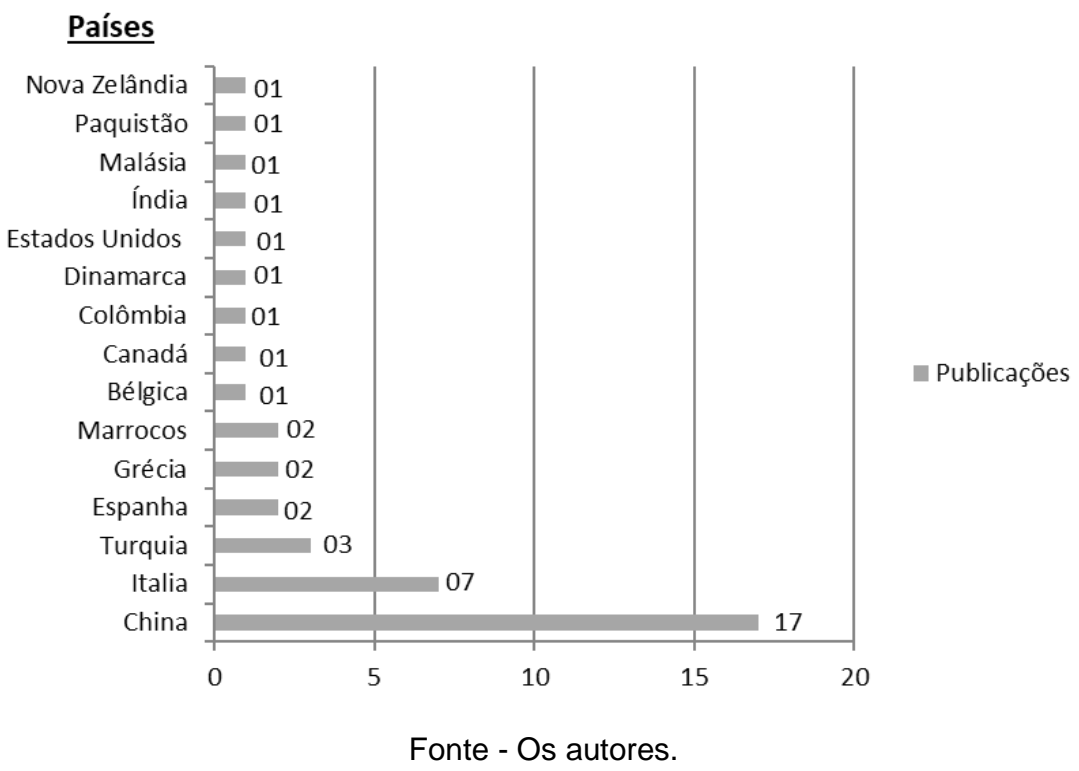




\section{ANÁLISE DOS RESULTADOS}

Esta seção visa discutir a partir dos trabalhos selecionados as propostas de integração loT e GIS. Os resumos foram apresentados por área de aplicação, destacando as principais descobertas, vantagens e desvantagens em suas implementações.

\section{Resumo das Aplicações em segurança}

Huang et al. (2010) oferecem uma solução completa e prática para localização de vítimas em caso de desastres em mineradoras subterrâneas. O sistema é baseado em uma rede de transmissão ZigBee, Ethernet com visualização em WebGIS e algoritmo baseado em Received Signal Strength Indicator (RSSI). A plataforma WebGIS é utilizada para desenvolver uma interface gráfica amigável, capaz de exibir a movimentação dos mineiros em tempo real. O módulo de análise e gravação de dados do servidor passam as informações para o banco de dados, e em seguida, exibe a distribuição de mineiros no mapa.

Chen et al. (2011) apresentam um sistema que combina loT e GIS para a estimativa de perdas econômicas e gerenciamento de resgates de vítimas oriundas de terremotos na China. O sistema utiliza a integração de sensores para coletar e monitorar os dados em tempo real quanto a movimentações geomorfológicas. Para este trabalho, os autores utilizaram dados vetoriais topográficos, informações quantitativas de população, localização das edificações, limites regionais e dados econômicos, ambos, armazenados em um SQL server e visualizado em ambiente GIS desktop. No âmbito da loT a rede de transmissão dos dados 3G e 4G com sensores de acelerômetro monitoram a superfície em questão.

$\mathrm{Na}$ busca de otimizar o gerenciamento quanto emergências por fenômenos hidrogeológicos Mangiamelli e Mussumeci (2013), aplicaram um sistema GIS-Spatial Data Base Management System (DBMS) para a criação de um WebGIS desenvolvido completamente por softwares livres. O sistema de baixo custo utiliza o Map Server, Pmapper, Apache, GRASS e PostGIS para a elaboração do banco de dados e desenvolvimento do WebGIS. Enquanto base de dados, os autores utilizam o Google Maps e o Open Street Map (OSM), além de dados obtidos por receptores do Global Position System (GPS), a transmissão dos dados é feita por rádio frequência em tempo real. A aplicação deste sistema se torna útil quando necessário transferir informações em curto espaço de tempo para o centro de operações e resgate de pessoas. Sua utilização otimiza significantemente o uso de homens e meios disponíveis para a prestação de assistência emergencial para as populações afetadas.

Pensando no desenvolvimento de planos de emergência para eventos catastróficos Huang et al. (2015), desenvolvem uma plataforma 3D baseada em WebGIS. A integração com a loT possibilita um sistema de alerta antecipado e em tempo real do fluxo de detritos em uma região de montanha na cidade de Quingping, província de Sichuan, China. O ambiente da plataforma fornece: (a) um banco de dados projetado para várias origens e combinações em uma única estrutura de servidor, (b) limites de precipitação armazenados no banco de dados para as ravinas envolvidas, (c) uma interface gráfica baseada em $3 D$ WebGIS fornecendo uma visualização realista e um sistema de fácil acesso para tomadores de decisão.

Luchettii et al. (2017), utilizam tecnologias como Augmented Reality (AR), Crowd Mapping (CM), Internet of Things (IoT), Wireless Sensor Networks (WSN) e o Geographic Information System (GIS) para prevenção e manejo correto acerca de desastres hidrogeológicos. Destaca-se, o sistema integrado composto por um Geo-server (GeoData Collector) que é responsável pela coleta e análise dos dados obtidos pelas redes sociais. O atual protótipo acessa a rede social do Twitter para busca de informações de eventos catastróficos e as disponibilizam em um aplicativo móvel por meio de uma Application Programming Interface (API).

Athanasiou et al. (2018) apresenta o desenvolvimento de um WebGIS que serve como mecanismo online para prevenção, monitoramento e alerta contra inundações, mais especificamente no Rio Arachthos, na Grécia. A pesquisa descreve brevemente as principais tecnologias para 0 desenvolvimento de um sistema GIS online, sendo eles: mapeamento de tecnologias, desenvolvimento de bancos de dados e aplicações web. O WebGIS desenvolvido combina dados provenientes de diferentes fontes, como por exemplo: pluviometria, temperatura, velocidade do vento e nível de água. O sistema que é composto por rede de sensores visa aprimorar o conhecimento do governo local e de interessados diretos, a fim de melhorar os processos de tomada de decisão e a gestão eficiente de crises associadas a eventos de inundação, além disso, manter a população informada em casos de emergências.

Jia et al. (2012) exploram modelos e mecanismos para uma aplicação segura quanto a produção, armazenamento e transporte de fogos de artifícios. Com o sistema baseado em tecnologias loT a

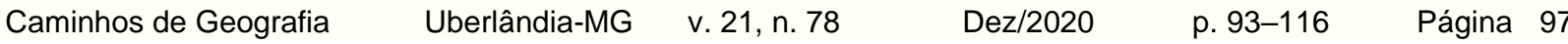


aplicação utiliza sensores de temperatura e umidade, além da detecção de tags via Rádio Frequency Identification (RFID), a fim de coletar dados que possam afetar segurança da matéria-prima do processo produtivo a entrega final.

Por meio de uma arquitetura conceitual Cherradi et al. (2017), propõe um sistema que possibilita a gestão de riscos quanto ao monitoramento para transporte de cargas perigosas, embasados em tecnologia GIS e loT. A utilização de uma rede Wireless Fidelity (WiFI) com protocolo Message Queue Telemetry Transport (MQTT) foi adotada para a transmissão dos dados de sensores em tempo real. Um módulo GPS, possibilita a identificação espacial e o armazenamento dos dados é orientado por meio do QGIS Server e PostGIS.

Ainda no mesmo ano, Cherradi et al. (2017), apresentam uma nova publicação com testes no sistema inicialmente desenvolvido. Desta vez, além de utilizarem apenas softwares de código aberto, criaram uma plataforma de visualização baseada na Web, utilizando como base de dados geográficos o Open Street Map (OSM). As informações do posicionamento dos veículos, bem como os dados dos sensores foram convertidos no formato GeoJSON para serem visualizadas nesta plataforma.

Thanos et al. (2016), apresentam o SYNAISTHISI, um aplicativo móvel para smartphones que oferece recursos de navegação precisa dentro e fora das edificações. O aplicativo contém um conjunto de serviços úteis, incluindo recomendações personalizadas, gerenciamento de visitas ou eventos e uma funcionalidade de pesquisa útil para navegar em tempo real de um local para outro. À medida que o usuário encontra seu caminho em direção ao seu destino, os pontos de verificação habilitados por Near-field Communication (NFC) e bluetooth o ajudam oferecendo recursos de reencaminhamento e informações úteis sobre reuniões em andamento e eventos que estejam acontecendo em sua proximidade. O aplicativo é suportado por um sistema GIS de back-end que possibilita o fornecimento de uma visão ampla e clara para os organizadores de evento, gerentes de campus universitários, equipes de campo, logística e segurança.

Wu et al. (2017), apresentam um sistema de monitoramento por vídeo e rastreamento de trajetória de pessoas para à gestão da segurança. A abordagem retrata o gerenciamento de dados em tempo real por meio de detecção de anormalidades. O sistema permite a organização e o gerenciamento de dados geoespaciais em tempo real e avanços em um amplo espectro de aplicações, no que tange principalmente, o uso de elementos geográficos unificados em um Sistema Gerenciador de Banco de Dados (SGBD) e via Structured Query Language (SQL). Além disso, usando o mecanismo de mensagem de assinatura e publicação para mapear as relações entre objetos geográficos, eventos e processos, o método demonstra praticidade e confiabilidade no suporte de aplicações GIS em tempo real.

Peng et al. (2017), desenvolvem um sistema para gerenciamento de emergências (não clínicas) em hospitais. Tal produção aplica uma integração entre IOT, BIM e GIS para a detecção de riscos, análise, visualização e tomada de decisão, por meio de uma arquitetura de sistema que utiliza sensores de temperatura, fumaça e qualidade do ar. Com os dados visualizados em tempo real, uma rede Local Area Network (LAN) foi desenvolvida para a comunicação interna e uma rede Wide Area Network (WAN) possibilita uma comunicação externa e mais abrangente, como por exemplo, com o corpo de bombeiros em casos de incêndio. O software GIS indicado para integração foi ArcGIS, desenvolvido pela Esri.

Os artigos discutidos destacam os seguintes temas: Monitoramento, prevenção e resgate em decorrência de fenômenos naturais (6), gestão da segurança para cargas perigosas (3), gestão da segurança pública (2), gestão de riscos emergenciais não clínicos em hospitais. Nestes, as principais vantagens apontadas estão associadas ao uso de softwares livres, com baixo custo de sistema e utilização de dados abertos (Quadro 1).

Quadro 1 - Síntese das aplicações em segurança.

\begin{tabular}{|c|c|c|c|c|c|}
\hline Refs. & $\begin{array}{c}\text { Ano da } \\
\text { Pesquisa }\end{array}$ & Objetivo & Vantagem & Desvantagem & Descoberta \\
\hline Liu et al & 2008 & $\begin{array}{c}\text { Implementação de um sistema } \\
\text { para monitoramento de transporte } \\
\text { urbano }\end{array}$ & $\begin{array}{c}\text { Flexibilidade } \\
\text { para explorar } \\
\text { diferenes tipos } \\
\text { de dados no } \\
\text { webgis }\end{array}$ & $\begin{array}{c}\text { Alto custo e } \\
\text { baixa } \\
\text { transmissão }\end{array}$ & $\begin{array}{c}\text { Arquitetura } \\
\text { e algorítimo }\end{array}$ \\
\hline Huang et al. & 2015 & $\begin{array}{c}\text { Apresenta uma proposta para } \\
\text { uma plataforama 3DWebGIS } \\
\text { combinada a uma rede de } \\
\text { sensores para monitoramento de } \\
\text { alerta de detritos de ravina. }\end{array}$ & $\begin{array}{c}\text { Aplicabilidade } \\
\text { prática }\end{array}$ & $\begin{array}{c}\text { Limitação de } \\
\text { memória para } \\
\text { transmissão de } \\
\text { dados e perda } \\
\text { de dados na }\end{array}$ & Plataforma \\
\hline \multicolumn{1}{|c|}{ Caminhos de Geografia } & Uberlândia-MG v. 21, n. 78 & Dez/2020 & p. 93-116 & Página 98 \\
\hline
\end{tabular}




\begin{tabular}{|c|c|c|c|c|c|}
\hline & & & & $\begin{array}{l}\text { integração de } \\
\text { sensores }\end{array}$ & \\
\hline Thanos et al. & 2016 & $\begin{array}{l}\text { Criar um aplicativo móvel para } \\
\text { smartphones que ofereça } \\
\text { recursos de localização dentro e } \\
\text { fora de edificações }\end{array}$ & $\begin{array}{c}\text { Uso de software } \\
\text { livre, } \\
\text { personalização } \\
\text { dinâmica e } \\
\text { detalhada, } \\
\text { môdulo de } \\
\text { análise de } \\
\text { opnião dos } \\
\text { usuários }\end{array}$ & Não discutido & $\begin{array}{l}\text { Aplicativo, } \\
\text { plataforma } \\
e \\
\text { algorítimos }\end{array}$ \\
\hline Sciortino & 2016 & $\begin{array}{l}\text { Aplicação de tecnologias para } \\
\text { monitoramento do transporte de } \\
\text { produtos perecíveis }\end{array}$ & $\begin{array}{l}\text { Modelo permite } \\
\text { um bom } \\
\text { monitoramento } \\
\text { e controle }\end{array}$ & $\begin{array}{l}\text { Alto custo de } \\
\text { implementação }\end{array}$ & $\begin{array}{l}\text { Protótipo, } \\
\text { algorítimo e } \\
\text { plataforma }\end{array}$ \\
\hline Peng et al. & 2017 & $\begin{array}{c}\text { Apresenta um sistema de } \\
\text { gerenciamento de risco em } \\
\text { hospitais integrando loT, BIM e } \\
\text { GIS }\end{array}$ & Baixo custo & $\begin{array}{l}\text { Não há uma } \\
\text { padronização } \\
\text { dos alarmes } \\
\text { quanto aos } \\
\text { diferentes tipos } \\
\text { alertas }\end{array}$ & $\begin{array}{l}\text { Arquitetura } \\
\text { de sistema }\end{array}$ \\
\hline $\begin{array}{l}\text { Luchettii et } \\
\text { al. }\end{array}$ & 2017 & $\begin{array}{c}\text { Fazer um uso integrado da } \\
\text { Realidade Aumentada (RA), } \\
\text { Crowd Mapping (CM), Internet of } \\
\text { Things (IOT), Wireless Sensor } \\
\text { Networks (WSN) e Geographic } \\
\text { Informations System (GIS) para } \\
\text { tomada de decisões acerca de } \\
\text { desastres naturais. }\end{array}$ & $\begin{array}{c}\text { Uso de } \\
\text { softwares livre, } \\
\text { servidor leve } \\
\text { com baixo } \\
\text { consumo de } \\
\text { dados, baixo } \\
\text { esforço } \\
\text { computacional }\end{array}$ & $\begin{array}{l}\text { Limitações na } \\
\text { quantidade de } \\
\text { pesquisa de } \\
\text { ocorrências } \\
\text { junto a rede } \\
\text { social utilizada }\end{array}$ & Protótipo \\
\hline Wu et al. & 2017 & $\begin{array}{l}\text { Abordagem aplicada a segurança } \\
\text { pública para uma organização e } \\
\text { gerenciamento de dados } \\
\text { geoespaciais de grande volume } \\
\text { em tempo real }\end{array}$ & $\begin{array}{l}\text { Estabilidade na } \\
\text { transferência de } \\
\text { dados }\end{array}$ & Não discutido & Framework \\
\hline $\begin{array}{l}\text { Athanasiou } \\
\text { et al. }\end{array}$ & 2018 & $\begin{array}{l}\text { Apresentar um WebGIS para } \\
\text { monitoramento e alertas contra } \\
\text { inundações }\end{array}$ & $\begin{array}{l}\text { Uso de dados } \\
\text { abertos }\end{array}$ & Não discutido & $\begin{array}{l}\text { Arquitetura } \\
\text { de sistema }\end{array}$ \\
\hline $\begin{array}{l}\text { Gunduz et } \\
\text { al. }\end{array}$ & 2017 & $\begin{array}{c}\text { Apresentar e testar uma } \\
\text { arquitetura de software BIM, GIS } \\
\text { e IOT para apoiar análise de } \\
\text { conforto. }\end{array}$ & $\begin{array}{l}\text { Aumento da } \\
\text { eficiência no } \\
\text { gerenciamento } \\
\text { das instalações }\end{array}$ & $\begin{array}{c}\text { Poucos } \\
\text { estudos para } \\
\text { com esse tipo } \\
\text { de escopo }\end{array}$ & $\begin{array}{l}\text { Arquitetura } \\
\text { de sistema }\end{array}$ \\
\hline Nourjou & 2017 & $\begin{array}{l}\text { Apresentar um sistema baseado } \\
\text { na web em tempo real }\end{array}$ & $\begin{array}{l}\text { Plataforma } \\
\text { flexível }\end{array}$ & Alto custo & $\begin{array}{c}\text { Arquitetura } \\
\text { de sistema } \\
e \\
\text { plataforma }\end{array}$ \\
\hline Bottacciloli & 2017 & $\begin{array}{c}\text { Desenvolver uma plataforma } \\
\text { integrada em tempo real para } \\
\text { dados de simulação de energia } \\
\text { fotovoltaica }\end{array}$ & $\begin{array}{l}\text { Plataforma } \\
\text { flexível }\end{array}$ & Alto custo & Plataforma \\
\hline Bottacciloli & 2017 & $\begin{array}{c}\text { Apresentar uma arquitetura para } \\
\text { simulação e modelagem de } \\
\text { desempenho energético em } \\
\text { edifícios. }\end{array}$ & $\begin{array}{l}\text { Rede de } \\
\text { sensores } \\
\text { otimizada }\end{array}$ & Alto custo & $\begin{array}{l}\text { Arquiterura } \\
\text { de sistema }\end{array}$ \\
\hline Li et al. & 2017 & $\begin{array}{l}\text { Projetar um sistema de inspeção } \\
\text { eletrônica com o uso de RFID e } \\
\text { sensores web. }\end{array}$ & $\begin{array}{l}\text { Baixo custo e } \\
\text { inspeção sem } \\
\text { contato }\end{array}$ & $\begin{array}{l}\text { Necessidade } \\
\text { de testes com } \\
\text { maior número } \\
\text { de sensores }\end{array}$ & $\begin{array}{l}\text { Arquiterura } \\
\text { de sistema }\end{array}$ \\
\hline Hamide & 2017 & $\begin{array}{l}\text { Avaliar diferentes tipos de } \\
\text { sistemas inteligentes para a } \\
\text { criação de um sistema de } \\
\text { estacionamento inteligente. }\end{array}$ & $\begin{array}{l}\text { Sensores } \\
\text { inteligentes } \\
\text { possibilitam a } \\
\text { otimização } \\
\text { espacial }\end{array}$ & Alto custo & Protótipo \\
\hline $\begin{array}{l}\text { Jagwani e } \\
\text { Kumar }\end{array}$ & 2018 & $\begin{array}{l}\text { Propõe uma estrutura baseada na } \\
\text { web para rastreamento de frotas } \\
\text { em tempo real }\end{array}$ & $\begin{array}{l}\text { Baixo custo, } \\
\text { interface } \\
\text { amigável, } \\
\text { sistema estável } \\
\text { e robusto }\end{array}$ & $\begin{array}{l}\text { baixa precisão } \\
\text { do sinal GPS }\end{array}$ & Protótipo \\
\hline
\end{tabular}

Fonte - Os autores. 


\section{Resumo das Aplicações em operação e manutenção}

Os estudos continuados de Gunduz et al. 2017, testam uma arquitetura de softwares para a integração de Building Information Modeling (BIM), GIS e loT que possibilite a análise de conforto e supere os problemas quanto ao gerenciamento de instalações complexas. Tal abordagem utiliza um modelo BIM convertido e exibido em 2D no Google Maps com dados dos sensores vistos em tempo real. Sua visualização ocorre por meio de plataforma de prototipagem eletrônica (Arduino) e sensores que disponibilizam informações de umidade, temperatura e nível de luz da edificação.

Os autores buscam que a integração destas tecnologias apresente um impacto significativo na solução de problemas na gestão de Facilities. Para eles, o BIM, IoT e o GIS integrados podem auxiliar na investigação e relação dinâmica entre as condições conforto e geometria das edificações. Tal sistema, visa melhorar o planejamento urbano e o projeto de construção em termos de otimização de energia.

Nourjou e Hashemipour (2017), apresentam um sistema que permite que aplicativos habilitados para mapeamento geográfico (como mapeamento da web, GIS para dispositivos móveis, painel de operações) acessem, consultem e exibam mapas na web associado ao status de interrupção de energia e informações de perda econômica em tempo real no estado da Flórida, nos Estados Unidos.

O sistema inclui o serviço Warehouse Management System (WMS) com via Localizador Padrão de Recursos (URL) definida para compartilhar possíveis interrupções quanto ao serviço em tempo real. Em seguida, os autores criaram aplicativos de mapeamento na web usando o ArcGIS. Os resultados do sistema apontam uma para plataforma flexível que podem também ser aplicada no gerenciamento de operação e manutenção, cidades inteligentes e emergências. Neste, os autores sinalizam elevado custo para a implementação sistema, em consequência do uso de ferramentas privadas.

Bottacciloli et al. (2017), expõe uma plataforma integrada visualizada em tempo real que simula a distribuição de energia fotovoltaica em um distrito de Turim, na Itália. A ferramenta permite simular a potência produzida por geradores fotovoltaicos em telhados de edifícios sob condições reais, além de analisar o comportamento da rede quanto a geração e consumo. Os dois componentes são interconectados pela internet através de um adaptador de software de comunicação. Os testes apontam para uma plataforma que pode ser útil no futuro para analisar diferentes cenários de penetração solar e testes quanto a novos procedimentos operacionais para a rede de distribuição.

Em uma outra pesquisa, Bottacciloli et al. (2017), desenvolve uma arquitetura na nuvem que integra componentes de hardware e software para monitorar e modelar o comportamento de energia em edifícios. A plataforma explora dispositivos loT georreferenciados para coletar informações relacionadas ao consumo de energia em uma edificação. Os dispositivos loT são integrados a tecnologias de middleware por conversão de dados BIM (formato gbXML), GIS e dados meteorológicos. Permitindo assim, uma boa precisão quanto às simulações de energia. Além disso, os dados fornecidos pelos dispositivos loT podem ser usados também para monitoramento quase em tempo real e o controle dos parâmetros ambientais e de energia dos edifícios.

Li et al. (2017), propõe um estudo para modernização quanto ao processo de inspeção em linhas de transmissão de energia elétrica. O sistema testado permite inteligência na inspeção de equipamentos eletrônicos de potência com o uso de Radio-Frequency IDentification (RFID), multi-sensores em tempo real e por meio de uma rede de transmissão $3 G, 4 G$ e WiFi.

De modo geral, os artigos selecionados para uma aplicação integrada em operação e manutenção traz produções divididas em dois grupos: Facilities management (1) e gestão da energia (4). Os artigos se destacam em consequência do desenvolvimento de novas arquiteturas de sistemas que favoreçam a integração loT e GIS (Quadro 2). 
Quadro 2 - Síntese das aplicações em operação e manutenção.

\begin{tabular}{|c|c|c|c|c|c|}
\hline Refs. & $\begin{array}{c}\text { Ano da } \\
\text { Pesquisa }\end{array}$ & Objetivo & Vantagem & Desvantagem & Descoberta \\
\hline Gunduz et al. & 2017 & $\begin{array}{c}\text { Apresentar e testar uma } \\
\text { arquitetura de software BIM, } \\
\text { GIS e IOT para apoiar } \\
\text { análise de conforto. }\end{array}$ & $\begin{array}{c}\text { Aumento da } \\
\text { eficiência no } \\
\text { gerenciamento } \\
\text { das } \\
\text { instalações }\end{array}$ & $\begin{array}{c}\text { Poucos estudos } \\
\text { para com esse } \\
\text { tipo de escopo }\end{array}$ & $\begin{array}{c}\text { Arquitetura } \\
\text { de sistema }\end{array}$ \\
\hline Nourjou & 2017 & $\begin{array}{c}\text { Apresentar um sistema } \\
\text { baseado na web em tempo } \\
\text { real }\end{array}$ & $\begin{array}{c}\text { Plataforma } \\
\text { flexível }\end{array}$ & Alto custo & $\begin{array}{c}\text { Arquitetura } \\
\text { de sistema e } \\
\text { plataforma }\end{array}$ \\
\hline Bottacciloli & 2017 & $\begin{array}{c}\text { Desenvolver uma } \\
\text { plataforma integrada em } \\
\text { tempo real para dados de } \\
\text { simulação de energia } \\
\text { fotovoltaica }\end{array}$ & $\begin{array}{c}\text { Plataforma } \\
\text { flexível }\end{array}$ & Alto custo & Plataforma \\
\hline Bottacciloli & 2017 & $\begin{array}{c}\text { Apresentar uma arquitetura } \\
\text { para simulação e } \\
\text { modelagem de desempenho } \\
\text { energético em edifícios. }\end{array}$ & $\begin{array}{c}\text { Rede de } \\
\text { sensores } \\
\text { otimizada }\end{array}$ & Alto custo & $\begin{array}{c}\text { Arquiterura } \\
\text { de sistema }\end{array}$ \\
\hline $\begin{array}{c}\text { Projetar um sistema de } \\
\text { inspeção eletrônica com o } \\
\text { uso de RFID e sensores } \\
\text { web. }\end{array}$ & $\begin{array}{c}\text { Baixo custo e } \\
\text { inspeção sem } \\
\text { contato }\end{array}$ & $\begin{array}{c}\text { Necessidade de } \\
\text { testes com } \\
\text { maior número } \\
\text { de sensores }\end{array}$ & $\begin{array}{c}\text { Arquiterura } \\
\text { de sistema }\end{array}$ \\
\hline
\end{tabular}

Fonte - Os autores.

\section{Resumo das Aplicações em saúde}

Tan et al. (2012), apresentam o Emergency Medical Service System (RTIEMS), um sistema de atendimento médico de emergência baseado em redes de comunicação e integrado a uma plataforma WebGIS. Esta proposta é baseada na instalação de câmeras e sensores biomédicos que transmitem em tempo real as informações do paciente para o hospital. Além disso, o sistema proposto possibilita a redução no tempo do resgate, uma vez, que utiliza receptores GPS e algoritmos para cálculo de rotas mais próximas e menos congestionadas. A visualização web utiliza imagens de satélites disponíveis na base de dados do Google Maps, o armazenamento das informações em banco de dados SQL e o sistema de comunicação baseado em rede wireless.

Marek et al. (2016) demonstram um projeto piloto onde visam analisar a influência da qualidade do ar para a saúde de pessoas que sofrem com doença pulmonar obstrutiva crônica. Os pacientes utilizam um aplicativo vinculado a um dispositivo GPS no Smartphone. Com essas informações conectadas a dados de qualidade do ar, pode-se estimar a exposição e a vulnerabilidade do paciente com mais precisão. A ideia dos autores é de conhecer os movimentos diários dos pacientes em tempo real e que estas intervenções a longo prazo possam levar a mudanças drásticas quanto a qualidade de vida destes pacientes.

No contexto das aplicações associadas a saúde destacam-se trabalhos para monitoramento para redução do tempo em atendimentos de emergência e influência da qualidade do ar na vida das pessoas. Nestes a síntese destaca a possibilidade destas aplicações para um sistema de baixo custo e com a utilização de dados abertos (Quadro 3).

Quadro 3 - Síntese das aplicações em saúde.

\begin{tabular}{|c|c|c|c|c|c|}
\hline Refs. & $\begin{array}{c}\text { Ano da } \\
\text { Pesquisa }\end{array}$ & Objetivo & Vantagem & Desvantagem & Descoberta \\
\hline Tan et al. & 2012 & $\begin{array}{c}\text { Apresentar um sistema de } \\
\text { atendimento médico } \\
\text { baseado em informações } \\
\text { em tempo real }\end{array}$ & $\begin{array}{c}\text { Uso de dados } \\
\text { abertos }\end{array}$ & Não discutido & $\begin{array}{c}\text { Arquitetura de } \\
\text { sistema e } \\
\text { algoritmo }\end{array}$ \\
\hline Marek et al. & 2016 & $\begin{array}{c}\text { Analisar a influência da } \\
\text { qualidade do ar na saúde } \\
\text { de pessoas que sofrem } \\
\text { com doença pulmonar } \\
\text { obstrutiva crônica }\end{array}$ & Baixo custo & $\begin{array}{c}\text { Monitoramento } \\
\text { quase em } \\
\text { tempo real }\end{array}$ & $\begin{array}{c}\text { Ecossistema de } \\
\text { dados }\end{array}$ \\
\hline
\end{tabular}

Fonte - Os autores. 


\section{Resumo das Aplicações em Tráfego e Mobilidade Urbana}

Nas amostras destinadas a aplicação de tráfego e mobilidade urbana, detectou-se as seguintes linhas de pesquisa: monitoramento e rastreamento de veículos (5) e estacionamento inteligente (1). De acordo com Liu et al. (2008), Sciortino (2016) e Hamide (2017) alguns destes artefatos apresentam um custo elevado, o que a depender do tipo de projeto pode significar um impasse para sua viabilidade (Quadro 4).

Quadro 4 - Síntese das aplicações em tráfego e mobilidade

\begin{tabular}{|c|c|c|c|c|c|}
\hline Refs. & $\begin{array}{c}\text { Ano da } \\
\text { Pesquisa }\end{array}$ & Objetivo & Vantagem & Desvantagem & Descoberta \\
\hline Liu et al. & 2008 & $\begin{array}{l}\text { Implementação de um } \\
\text { sistema para } \\
\text { monitoramento de } \\
\text { transporte urbano }\end{array}$ & $\begin{array}{c}\text { Flexibilidade } \\
\text { para explorar } \\
\text { diferentes } \\
\text { tipos de dados } \\
\text { no Webgis }\end{array}$ & $\begin{array}{l}\text { Alto custo e baixa } \\
\text { transmissão }\end{array}$ & $\begin{array}{l}\text { Arquitetura e } \\
\text { algorítimo }\end{array}$ \\
\hline Pirotti et al. & 2011 & $\begin{array}{c}\text { Metodologia para } \\
\text { implementação de uma } \\
\text { estrutura eficiente baseada } \\
\text { na web para } \\
\text { monitoramento quanto a } \\
\text { riscos de acidentes com } \\
\text { veículos }\end{array}$ & $\begin{array}{l}\text { Uso de dados } \\
\text { colaborativos, } \\
\text { análises } \\
\text { automatizadas }\end{array}$ & Não discutido & $\begin{array}{c}\text { Arquiterura e } \\
\text { Framework }\end{array}$ \\
\hline Gan & 2015 & $\begin{array}{c}\text { Desenvolve um método } \\
\text { para rastreamento de } \\
\text { cargas em tempo real, } \\
\text { baseado em tecnologias } \\
\text { loT. }\end{array}$ & $\begin{array}{l}\text { Identificação } \\
\text { automatizada } \\
\text { dos produtos, } \\
\text { monitoramento } \\
\text { integrado }\end{array}$ & Não discutido & $\begin{array}{c}\text { Arquitetura } \\
\text { de sistema e } \\
\text { plataforma }\end{array}$ \\
\hline Sciortino & 2016 & $\begin{array}{c}\text { Aplicação de tecnologias } \\
\text { para monitoramento do } \\
\text { transporte de produtos } \\
\text { perecíveis }\end{array}$ & $\begin{array}{l}\text { Modelo } \\
\text { permite um } \\
\text { bom } \\
\text { monitoramento } \\
\text { e controle }\end{array}$ & $\begin{array}{l}\text { Alto custo de } \\
\text { implementação }\end{array}$ & $\begin{array}{l}\text { Protótipo, } \\
\text { algorítimo e } \\
\text { plataforma }\end{array}$ \\
\hline Hamide & 2017 & $\begin{array}{l}\text { Avaliar diferentes tipos de } \\
\text { sistemas inteligentes para } \\
\text { a criação de um sistema } \\
\text { de estacionamento } \\
\text { inteligente. }\end{array}$ & $\begin{array}{l}\text { Sensores } \\
\text { inteligentes } \\
\text { possibilitam a } \\
\text { otimização } \\
\text { espacial }\end{array}$ & Alto custo & Protótipo \\
\hline $\begin{array}{l}\text { Jagwani e } \\
\text { Kumar }\end{array}$ & 2018 & $\begin{array}{l}\text { Propõe uma estrutura } \\
\text { baseada na web para } \\
\text { rastreamento de frotas em } \\
\text { tempo real }\end{array}$ & $\begin{array}{l}\text { Baixo custo, } \\
\text { interface } \\
\text { amigável, } \\
\text { sistema } \\
\text { estável e } \\
\text { robusto }\end{array}$ & $\begin{array}{l}\text { baixa precisão do } \\
\text { sinal GPS }\end{array}$ & Protótipo \\
\hline
\end{tabular}

Fonte - Os autores.

Liu et al. (2008) discutem a implementação do Shenzhen Urban Transport Simulation System (SUTSS), expondo algoritmos para o cálculo e correspondência de mapas de velocidade instantânea do segmento rodoviário baseado em posicionamento Global Navigation Satellite Systems (GNSS). O sistema que é visualizado em plataforma WebGIS e utiliza cerca de 4000 motoristas de táxis enquanto sensores. Nos veículos foram embarcados receptores do Global Position System (GPS), conectados via wireless que transmitiam em intervalos regulares as informações para um servidor de dados. Mediante isso é possível calcular com exatidão as velocidades médias deste segmento rodoviário, pois as posições GPS dos táxis podem ser analisadas em termos de trajetórias de rota e velocidade, servindo como ferramenta para a gestão do tráfego e mobilidade urbana na China. 
Pirotti et al. (2011), apresentam uma plataforma de integração geográfica baseada na web para monitoramento quanto a riscos de acidentes na Itália. Um portal WebGIS foi criado para combinar um conjunto de informações (por exemplo: dados de acidentes e condições de tráfego) de interesse para uma avaliação segura do trânsito. Dados vetoriais, matriciais e de sensores comporão o conjunto de informações a serem analisadas. A utilização do MapServer, PostreSQL e PostGIS estão relacionadas para a elaboração do sistema WebGIS proposto.

Gan (2015) apresenta uma solução que integra tecnologias RFID, GPS, 3G e GIS para rastreamento e monitoramento em tempo real de mercadorias. Com base na solução proposta, a Internet of Things (loT) apresenta-se como uma boa tecnologia para rastreamento e serviços de logística. A tecnologia proposta oferece não apenas o status da remessa na forma de carregamento, descarregamento, partida, chegada e rota, mais também, uma localização geográfica detalhada sobre os pedidos. Para o autor, a solução ajuda a melhorar o planejamento de logística, controle de cargas, tomada de decisão por parte do cliente.

Sciortino et al. (2016), propõe um sistema técnico para monitoramento e controle da cadeia de produtos perecíveis. Os resultados da pesquisa apontam para uma previsão quanto a vida útil de um alimento durante o transporte logístico. Em particular, um WebGIS foi desenvolvido para o monitoramento em tempo real quanto ao prazo de validade destes alimentos.

Na Índia, Jagwani e Kumar (2018), propõe uma estrutura baseada na web para rastreamento de frotas através de um sistema flexível e totalmente personalizável, que além da localização em tempo real pode relatar parâmetros como velocidade do veículo, endereço geocodificado, histórico de localização. O sistema desenvolvido é baseado em tecnologias de comunicação como o General Packet Radio Services (GPRS), Global System for Mobile Communications (GSM), internet, Global Position System (GPS) e microcontroladores.

Em consequência da grande demanda por mobilidade urbana na Malásia, Hamide et al. (2017) avaliam diferentes tipos de sistemas inteligentes para a criação do goNpark. Esta tecnologia está sendo desenvolvida através do estudo de diferentes tipos de sistemas inteligentes de estacionamento e tecnologias para detecção de veículos. Os clientes serão capazes de obter a visualização em tempo real quanto a ocupação do estacionamento de destino, escolhendo assim a melhor localização ou o estacionamento mais próximo com o seu destino. O referido sistema envolve os seguintes módulos de funcionamento: (a) módulo de detecção de ocupação, (b) módulo de entrada e saída e (c) módulo de interface gráfica com o usuário.

\section{Resumo das Aplicações em Arquiteturas para Implementação}

Tang et al. (2010), combinam tecnologia Radio-Frequency IDentification (RFID) e Wireless Sensor Networks (WSN) para a elaboração de uma rede integrada a sensores e tecnologia GIS. Através do algoritmo Received Signal Strength Indication (RSSI) integrado a Tags, o sistema pode computar a localização de informações ambientais através de um mapa digital disponível em tempo real.

Yuan e Zhao (2012) propõe a criação de uma arquitetura para armazenamento de dados espaciais em ambiente Internet of Things (IOT), através de uma Spatial Data Warehouse System in IOT Environments (SDWIT), projetando os principais detalhes quanto a coleta, transmissão, armazenamento e atualização de dados. Nesta pesquisa, os autores justificam a necessidade de atualização do sistema operacional Spatial Data Warehouse (SDW) para o SDWIT por considerarem que o sistema anterior estaria ultrapassado para transmitir dados em tempo real provenientes de sensores.

Isiktag (2015) desenvolveu um método para facilitar a fusão de informações provenientes de dados do Building Information Modeling (BIM) e da Internet of Things (IOT), utilizando o Geographic Information System (GIS) como plataforma de visualização integrada destas informações. A utilização de modelos BIM e as informações adquiridas pelos sensores virtuais são integradas por meio da a utilização de serviços web e uma API RESTful baseada na nuvem (Amazon, Microsoft ou Google). Tal arquitetura possibilita a visualização destes dados em ambiente desktop ou web. Isiktag e Pilouk (2016) desenvolveram um experimento para uma arquitetura de aquisição de informação em tempo real a partir de feeds de Geo-sensores, utilização de web services, banco de dados gráficos para armazenamento e serviços GeoEvent no ArcGIS. A arquitetura descrita é testada com alguns sensores reais e virtuais. O experimento de integração é projetado e realizado em 4 etapas. A primeira etapa consistia em nós de loT reais e virtuais, a segunda é formada por um banco de dados de gráficos Neo4J. Na terceira, os componentes flexíveis desenvolvidos ajudaram na aquisição de informações do banco de dados gráfico e na representação dessas informações no formato

$\begin{array}{lllll}\text { Caminhos de Geografia } \quad \text { Uberlândia-MG } & \text { v. 21, n. 78 } & \text { Dez/2020 } & \text { p. 93-116 Página } 103\end{array}$


GeoJSON. A quarta é descrita pelo serviço GeoEvent, que é capaz de consumir feeds GeoJSON e representar as informações fornecidas pelos nós loT em tempo real.

Kiavarz et al. (2018), propuseram abordagem voltada a mineração, análise e interpretação de dados BIM, GIS e IOT, objetivando fornecer um mecanismo inteligente que facilite sua fusão. Neste artigo, os autores justificam adoção do conceito do Data Lake, em consequência da grande escala de dados atrelados a este tipo de integração a nível de cidade. No ambiente Data Lake os dados semiestruturados como nos formatos Industry Foundation Class (IFC), City Geography Markup Language (CityGML) ou extraídos dos sensores por formatos XML ou CSV, podem ser transferidos para um ambiente estruturado por meio de leitura de máquina, visualizações estatísticas, análise espacial e prescrição analítica. Os autores visam direcionar tal metodologia para linha Smart, como a Smart Building, Smart Citizens e a Smart Managenent, integrando diretamente dados 3D GIS, BIM e sensores. As literaturas apontam descobertas para arquiteturas de implementação envolvendo o desenvolvimento de plataformas, framework, sistemas e algoritmos.

Quadro 5 - Síntese das aplicações em arquiteturas de implementação.

\begin{tabular}{|c|c|c|c|c|c|}
\hline Refs. & $\begin{array}{c}\text { Ano da } \\
\text { Pesquisa }\end{array}$ & Objetivo & Vantagem & Desvantagem & Descoberta \\
\hline Tang et al. & 2010 & $\begin{array}{c}\text { Apresentar uma } \\
\text { plataforma de } \\
\text { identificação WSID } \\
\text { baseada em RFID e } \\
\text { WSN }\end{array}$ & Rede heterogêna & Não discutido & $\begin{array}{c}\text { Plataforma e } \\
\text { algorítimo }\end{array}$ \\
\hline Yuan et al. & 2012 & $\begin{array}{l}\text { Criação de um } \\
\text { sistema GIS e IOT } \\
\text { baseado em } \\
\text { SDWIT. }\end{array}$ & $\begin{array}{c}\text { Uso para aplicações } \\
\text { combinadas }\end{array}$ & Não discutido & Framework \\
\hline Isiktag & 2015 & $\begin{array}{l}\text { Desenvolvimento } \\
\text { de metodologia que } \\
\text { integra BiM, IOT e } \\
\text { GIS para auxílio em } \\
\text { gerenciamento }\end{array}$ & $\begin{array}{l}\text { Aplicável em qualquer } \\
\text { cliente GIS }\end{array}$ & $\begin{array}{c}\text { Necessita de } \\
\text { testes com } \\
\text { estudos de caso } \\
\text { reais }\end{array}$ & $\begin{array}{l}\text { Arquitetura } \\
\text { de sistema, } \\
\text { framework }\end{array}$ \\
\hline $\begin{array}{l}\text { Isiktag e } \\
\text { Pilouk }\end{array}$ & 2016 & $\begin{array}{c}\text { Abordar uma } \\
\text { arquitetura de } \\
\text { implementação } \\
\text { para } \\
\text { armazenamento, } \\
\text { exposição e } \\
\text { apresentação de } \\
\text { grande quantidade } \\
\text { de informações } \\
\text { geográficas } \\
\text { provinientes de } \\
\text { sensores em tempo } \\
\text { real. }\end{array}$ & $\begin{array}{c}\text { Compilação de nós loT } \\
\text { com sensores reais e } \\
\text { virtuais }\end{array}$ & Alto custo & $\begin{array}{l}\text { Arquitetura } \\
\text { de sistema }\end{array}$ \\
\hline $\begin{array}{l}\text { Kiavarz et } \\
\text { al. }\end{array}$ & 2018 & $\begin{array}{c}\text { Mineração de } \\
\text { dados 3D GIS e } \\
\text { BIM }\end{array}$ & $\begin{array}{l}\text { Possibilidade de fusão } \\
\text { entre dados 2D e 3D, } \\
\text { Definição de nós loT }\end{array}$ & Não discutido & Algorítimos \\
\hline
\end{tabular}

Fonte - Os autores.

\section{Resumo das Aplicações na Agricultura de Precisão}

Visando melhorias quanto a gestão hídrica em áreas agrícolas Soto-Garcia et al. (2013), desenvolveram um sistema baseado em webGIS para monitoramento em tempo real, ao qual facilite o acesso a água de modo eficiente para uma associação de agricultores em Cartagena, na Espanha. Sensores para medição de água, rede de transmissão wireless, dados de topografia cadastral, armazenamento em banco de dados SQL e Oracle, softwares como ArcGIS e AutocaMAP, envolveram o desenvolvimento do sistema. Os ganhos apresentados evolvem: (a) melhor eficiência 
da distribuição de água; (b) rastreabilidade da água; (c) economia de água; (d) gestão transparente; (e) redução quanto aos conflitos entre agricultores; (f) redução dos custos de consumo e energia.

Debauche et al. (2018), elabora um sistema de automação baseado IOT e GIS com a finalidade de melhorar a eficiência quanto ao uso da água, visando a otimização em processos de irrigação. A referida arquitetura permite armazenar e tratar dados em séries. Nesta pesquisa, atingiu-se a automação em tempo real para dados de umidade e temperatura com o uso de rede Wireless Sensors Network (WSN), juntamente com plataformas IOT em nuvem.

Cadvid et al. (2018), apresentam o Thingsboard, uma plataforma de código aberto usada para coletar e gerenciar dados fornecidos por uma hierarquia de dispositivos. Neste, a arquitetura proposta ilustra um monitoramento em tempo real a partir do uso de sensores de temperatura e umidade para controle de pragas.

Os contextos abordados nos trabalhos estão associados a temáticas que retratam aplicações visando um melhor gerenciamento da água e o monitoramento eficiente espécies. Nesta linha, a aplicação voltada para uma integração do loT e GIS é destacada pela necessidade de um alto investimento para sua implementação.

Quadro 6 - Síntese das aplicações em agricultura de precisão.

\begin{tabular}{|c|c|c|c|c|c|}
\hline Refs. & $\begin{array}{c}\text { Ano da } \\
\text { Pesquisa }\end{array}$ & Objetivo & Vantagem & Desvantagem & Descoberta \\
\hline Soto-Garcia et al. & 2013 & $\begin{array}{c}\text { Fornecer uma alocação } \\
\text { de água eficiente e } \\
\text { transparente a partir dos } \\
\text { usos das TIC's }\end{array}$ & $\begin{array}{c}\text { Monitoramento } \\
\text { eficiênte, acesso } \\
\text { aberto }\end{array}$ & Alto custo & Plataforma \\
\hline Debauche et al. & 2018 & $\begin{array}{c}\text { Propõe um sistema } \\
\text { automação baseado em } \\
\text { IOT e GIS para melhorar } \\
\text { a eficiência do uso da } \\
\text { água na agricultura }\end{array}$ & $\begin{array}{c}\text { Sistema } \\
\text { automatizado, } \\
\text { armazenamento } \\
\text { na nuvem }\end{array}$ & Alto custo & $\begin{array}{c}\text { Arquitetura } \\
\text { de sistema }\end{array}$ \\
\hline Cadavid et al. & 2018 & $\begin{array}{c}\text { Criar uma plataforma de } \\
\text { monitoramento que } \\
\text { permita a integração } \\
\text { entre loT, Data-Analytics } \\
\text { e especialistas em } \\
\text { espécies agrícolas. }\end{array}$ & $\begin{array}{c}\text { Uso de código } \\
\text { aberto, } \\
\text { armazenamento } \\
\text { na nuvem }\end{array}$ & Não discutido & $\begin{array}{c}\text { Plataforma } \\
\text { e algorítimo }\end{array}$ \\
\hline
\end{tabular}

Fonte - Os autores.

\section{Aplicações em Monitoramento Ambiental}

Pasquale et al. (2008) discute uma plataforma GIS que possibilite explorar, fundir e exibir dados ambientais a partir de uma Data Warehouse Virtual. Tal proposta visa a utilização dos dados de sensores ambientais obtidos por outras fontes para visualização em uma WebGIS em tempo real. As ferramentas e mecanismos incorporados nesta plataforma permitem uma rápida prototipagem para integrar de forma flexível várias visões do ambiente e fornecer previsões para a evolução dos parâmetros analisados quanto ao monitoramento da qualidade do ar.

Yuan et al. (2013), aponta para a necessidade da criação de um método de monitoramento ambiental que possa ser simples, rápido, aberto e com tecnologias de baixo custo. Para tanto, desenvolveram uma plataforma webGIS baseada em tecnologia Rich Internet Applications (RIA), para realizar o monitoramento em tempo real dos dados de temperatura, umidade, pluviometria, nível de água e velocidade do vento na China, a partir da rede de transmissão de rede wireless.

Gao et al. (2013), sinalizam a necessidade quanto ao desenvolvimento de uma plataforma para gerenciamento aberto, escalável e integrada para dar suporte ao serviço de troca ou recarga de baterias para veículos elétricos intermunicipais em grande escala. Neste sentido, apresentam um estudo abrangente para aplicação de loT na percepção de informação e transmissão associada a uma rede de veículos elétricos.

$\begin{array}{llllll}\text { Caminhos de Geografia } & \text { Uberlândia-MG } & \text { v. 21, n. } 78 & \text { Dez/2020 } & \text { p. 93-116 } & \text { Página } 105\end{array}$


O sistema informa aos usuários o momento para recarregamento de energia por meio de um lembrete de voz e os locais cujo condutor pode realizar o reabastecimento elétrico. Os terminais são interativos e inteligentes, pois utilizam sensores de energia da bateria e temperatura, além de um receptor GPS para exibir os parâmetros de localização e desempenho do veículo em um mapa.

Fang et al. (2014), discutem um sistema para monitoramento e gerenciamento de dados ambientais voltados a mudanças climáticas e baseados em loT e GIS. Os resultados da pesquisa indicam que o sistema introduzido é valioso para a percepção, transformação, processamento, gerenciamento e compartilhamento de informações de múltiplos recursos para monitoramento e auxílio na gestão ambiental.

Trellis et al. (2017), apresentam uma solução abrangente para fornecer dispositivos abertos para cidades inteligentes. A solução proposta mede as condições ambientais quanto aos parâmetros meteorológicos e de qualidade do ar em tempo real do Campus Universitário de Jaime I, Espanha. Os autores aplicam a integração dos sensores em ambiente GIS em uma plataforma aberta com software de origem proprietária.

An et al. (2014), apresentam uma proposta de um sistema de monitoramento remoto da qualidade da água baseada em IOT e tecnologia GPRS/3G. Para tanto, aplicam o processo em três níveis metodológicos (a) monitoramento do campo, (b) transmissão sem fio via wireless e (c) terminal móvel.

Mo et al. (2015), relatam a utilização de um sistema automático de monitoramento de água a partir de tecnologias loT. A aquisição e transmissão dos dados são realizadas por meio de sensores de vazão, volume, nível d'água e a comunicação com o banco de dados através de redes sem fio. Os dados são armazenados em banco Oracle e visualizados em WebGIS para interatividade e aplicações GIS no âmbito do monitoramento de recursos hídricos.

Para a melhoria da qualidade ambiental nas cidades é necessário que haja uma otimização quanto aos sistemas de coleta de lixo. Pensando nisso, Gutierrez et al. (2015) propõe um sistema para coleta de lixo inteligente. O protótipo identifica o nível de lixo existente nas lixeiras e possibilita um melhor gerenciamento quanto a coleta de resíduos urbanos. Os dados coletados pelos sensores (que utilizam placas Arduino) são enviados pela internet para um servidor, no qual são armazenados, processados e então usados para monitorar e otimizar a seleção diária de lixeiras a serem coletadas. Com base nesses dados, um processo de otimização permite criar as rotas de coleta mais eficientes, nas quais, são encaminhadas aos trabalhadores.

Draz et al. (2018), desenvolveram um sistema baseado em loT que permite a identificação em tempo real quanto ao estado de preenchimento das lixeiras. O sistema proposto identifica a localização de cada lixeira por meio de um módulo GPS e seu status de preenchimento é validado por meio se sensores volumétricos conforme Figura 4.

Figura 4 - Lixeiras inteligentes localizadas em Amsterdam e Bruxelas.
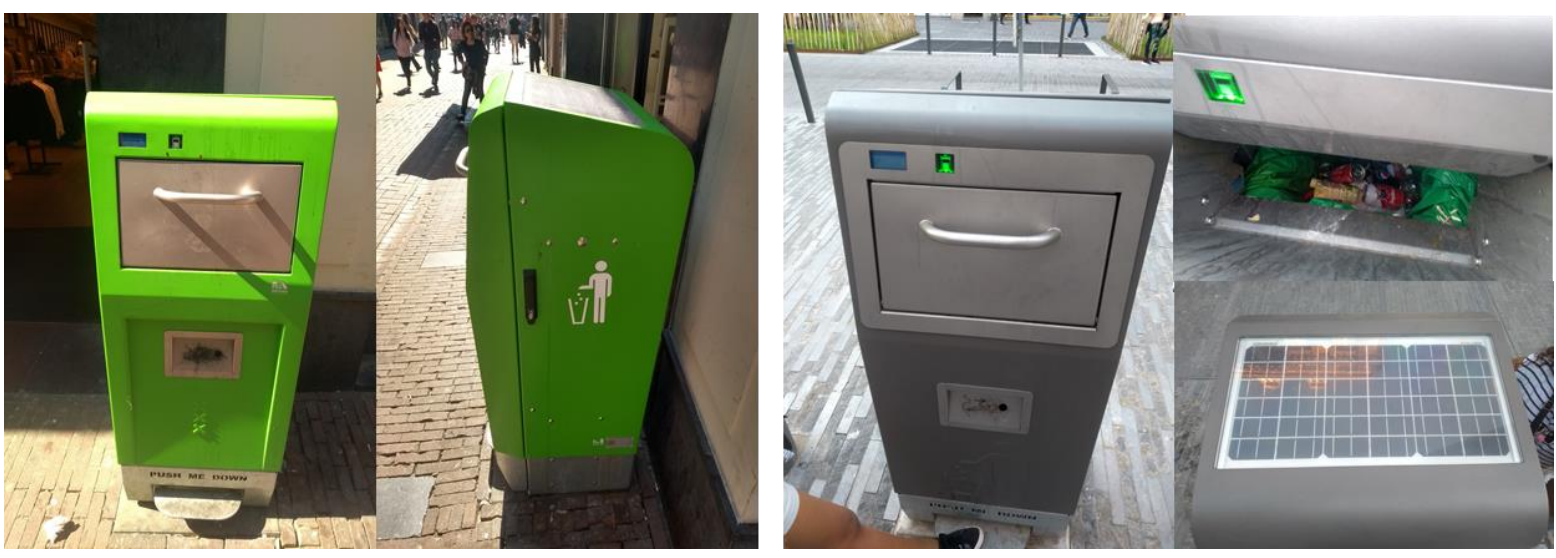

Fonte - Os autores.

Com o sinal enviado por uma rede Global System for Mobile Communications (GSM), o gestor municipal pode verificar as informações quanto a capacidade das lixeiras em qualquer lugar e a 
qualquer momento. Os autores indicam benefícios nesta aplicação, tais como: rotas otimizadas e precisas, uso eficaz das lixeiras, melhora da qualidade do ambiente.

Com base nas literaturas apresentadas a síntese das aplicações integradas apresentam as seguintes linhas gestão ambiental (5), gestão de recursos hídricos (2) e gerenciamento de resíduos sólidos (2). Vale salientar, que boa parte destas aplicações prezam pelo o uso de dados abertos, eficiência quanto a descoberta e tecnologias de baixo custo, conforme o Quadro 7.

Quadro 7 - Síntese das aplicações em monitoramento ambiental.

\begin{tabular}{|c|c|c|c|c|c|}
\hline Refs. & $\begin{array}{c}\text { Ano da } \\
\text { Pesquisa }\end{array}$ & Objetivo & Vantagem & Desvantagem & Descoberta \\
\hline Pasquale et al. & 2008 & $\begin{array}{c}\text { Apresenta uma plataforma } \\
\text { para exploração, fusão e } \\
\text { exibição de dados } \\
\text { ambientais por meio de } \\
\text { uma Virtual Data } \\
\text { Warehouse }\end{array}$ & $\begin{array}{l}\text { Acesso dos } \\
\text { dados por } \\
\text { diferentes } \\
\text { fontes }\end{array}$ & Não discutido & $\begin{array}{c}\text { Plataforma e } \\
\text { algorítimo }\end{array}$ \\
\hline Yuan et al. & 2013 & $\begin{array}{l}\text { Desenvolver um sistema } \\
\text { de baixo custo para } \\
\text { monitoramento e análise } \\
\text { de dados ambientais. }\end{array}$ & $\begin{array}{l}\text { Baixo custo, } \\
\text { código aberto }\end{array}$ & Não discutido & Plataforma \\
\hline Gao et al. & 2013 & $\begin{array}{l}\text { Instituir uma plataforma } \\
\text { para gerenciamento e } \\
\text { monitoramento de veiculos } \\
\text { elétricos em tempo real. }\end{array}$ & $\begin{array}{c}\text { Comunicação } \\
\text { colaborativa }\end{array}$ & $\begin{array}{l}\text { Rede de } \\
\text { comunicação } \\
\text { com baixa } \\
\text { cobertura, } \\
\text { plataforma } \\
\text { restrita }\end{array}$ & Plataforma \\
\hline Fang et al. & 2014 & $\begin{array}{c}\text { Apresentar um novo } \\
\text { sistema de integração de } \\
\text { monitoramento ambiental a } \\
\text { partir da integração da loT } \\
\text { e GIS. }\end{array}$ & $\begin{array}{l}\text { plataforma na } \\
\text { nuvem e } \\
\text { monitoramento } \\
\quad \text { eficaz }\end{array}$ & $\begin{array}{l}\text { Complexidade } \\
\text { para } \\
\text { integração de } \\
\text { dados de } \\
\text { múltiplas } \\
\text { fontes }\end{array}$ & Protótipo \\
\hline An et al. & 2014 & $\begin{array}{l}\text { Desenvolver um sistema } \\
\text { que integre dados de } \\
\text { sensores em tempo real } \\
\text { para monitoramento da } \\
\text { qualidade da água. }\end{array}$ & $\begin{array}{l}\text { Estável e } \\
\text { confiável }\end{array}$ & Não discutido & $\begin{array}{c}\text { Plataforma e } \\
\text { algorítimo }\end{array}$ \\
\hline Gutierrez et al. & 2015 & $\begin{array}{c}\text { Apresenta uma solução } \\
\text { para coleta de lixo } \\
\text { inteligente baseado em loT } \\
\text { e GIS. }\end{array}$ & $\begin{array}{l}\text { Uso de dados } \\
\text { abertos }\end{array}$ & Alto custo & Protóripo \\
\hline Mo et al. & 2015 & $\begin{array}{c}\text { Propor um sistema } \\
\text { integrado loT para gestão } \\
\text { de recursos hídricos. }\end{array}$ & $\begin{array}{l}\text { Monitoramento } \\
\text { eficaz e } \\
\text { confiável }\end{array}$ & Alto custo & Plataforma \\
\hline Trilles et al. & 2017 & $\begin{array}{c}\text { Criar uma plataforma } \\
\text { sensorizada que integre } \\
\text { loT e GIS para gestão } \\
\text { inteligente de campus } \\
\text { universitário, } \\
\text { especificamente focada a } \\
\text { dados ambientais }\end{array}$ & $\begin{array}{l}\text { Dados abertos, } \\
\text { baixo custo }\end{array}$ & Não discutido & Plataforma \\
\hline Draz et al. & 2018 & $\begin{array}{l}\text { Apresentar um sistema de } \\
\text { gerenciamento inteligente } \\
\text { para coleta de resíduos }\end{array}$ & Baixo custo & Não discutido & $\begin{array}{l}\text { Arquitetura de } \\
\text { sistema }\end{array}$ \\
\hline
\end{tabular}

Fonte - Os autores. 


\section{DISCUSSÃO}

Os resultados obtidos possibilitaram compreender o panorama atualizado acerca dos principais métodos para obtenção de dados espaciais, softwares utilizados, sensores, metodologias de armazenamento, plataformas de visualização, meios de transmissão e padrão dos dados utilizados no âmbito da integração loT e GIS.

O modelo de infraestrutura das camadas loT discutida por Gao, Zang e Li (2013), serviu de parâmetro teórico para a identificação e compilação. As camadas de percepção (constituída por sensores e plataformas de prototipagem eletrônica), transmissão (envolvendo acesso e transmissão em rede) e de aplicação (serviços web, saída de dados e observação dos dados), foram analisadas quantitativamente, vislumbrando assim, os principais tipos de sensores, as opções de rede para a transmissão dos dados e interface de visualização e atualização dos ciclos (Figura 5).

As aplicações na camada GIS são justificadas por seu potencial desenvolvido para a integração entre hardware e software, além das técnicas para captura, gerenciamento, análise e exibição dos dados geográficos (SOTO-GARCIA et al. 2015). Com o uso destes, é possível gerar relatórios gráficos automáticos e consultas rápidas por usuários não especialistas (SERRANO et al. 2008). Neste sentido, os principais blocos de investigação estão baseados nos tipos de dados utilizados, softwares para manipulação ou processamento dos dados, plataforma de armazenamento e visualização, além dos padrões e formatos destes dados.

\section{Principais camadas de domínio IOT}

No total das 42 publicações, cerca de $25 \%$ das pesquisas relacionam-se com a utilização dos sensores de temperatura e 17\% vinculadas aos sensores de umidade DHT11 ou DHT22 (Gráfico 1). As áreas de aplicação recorrentes a estas tecnologias foram: agricultura de precisão, segurança, monitoramento ambiental e operação e manutenção. As demais linhas de aplicação utilizaram a ampla gama de equipamentos disponíveis no mercado, como por exemplo, os sensores de qualidade do ar, energia, medidor de água, velocidade do vento, gás, dentre outros. Os percentuais de utilização destas pesquisas variaram entre $5 \%$ a $3 \%$.

Gráfico 1 - Relação Percentual x Quantitativo por camadas loT (Sensores).

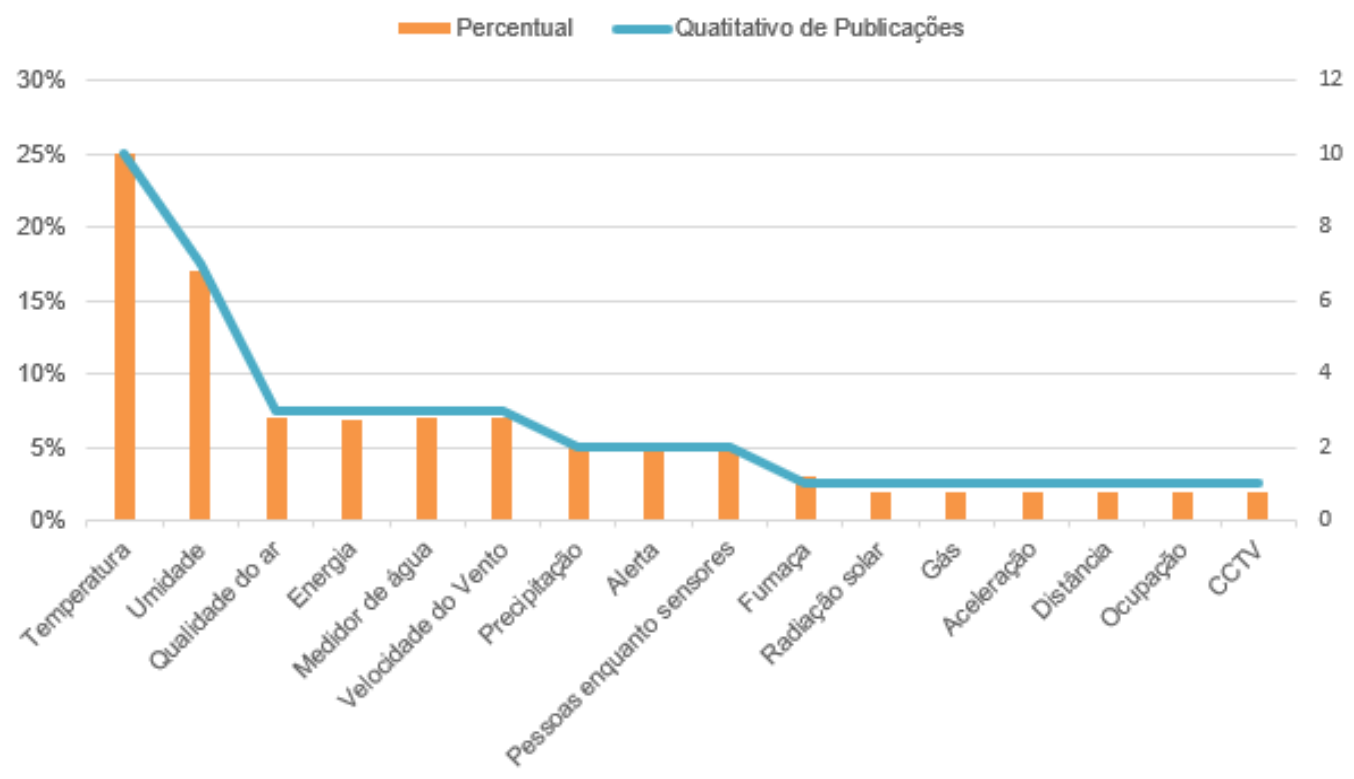

Fonte - Os autores.

As redes de transmissão 3G e General Packet Radio Service (GPRS) foram utilizadas em 18\% pesquisas. Nota-se um equilíbrio nos demais percentuais quanto a empregabilidade de outros métodos, como por exemplo, os protocolos Zigbee, rede 4G e Wireless Fidelity (Wifi). Essa relação é associada devido a adoção de critérios para a escolha de um método de transmissão que possibilite o atendimento da demanda. Este, de acordo com Debauche et al. (2018), deve levar em conta

$\begin{array}{lllll}\text { Caminhos de Geografia } \quad \text { Uberlândia-MG } & \text { v. 21, n. 78 } \quad \text { Dez/2020 } & \text { p. 93-116 Página } 108\end{array}$


principalmente o número de nós, distância e alcance necessário, altura da antena e a frequência de operação (Gráfico 2).

Gráfico 2 - Relação Percentual x Quantitativo por camadas loT (transmissão).

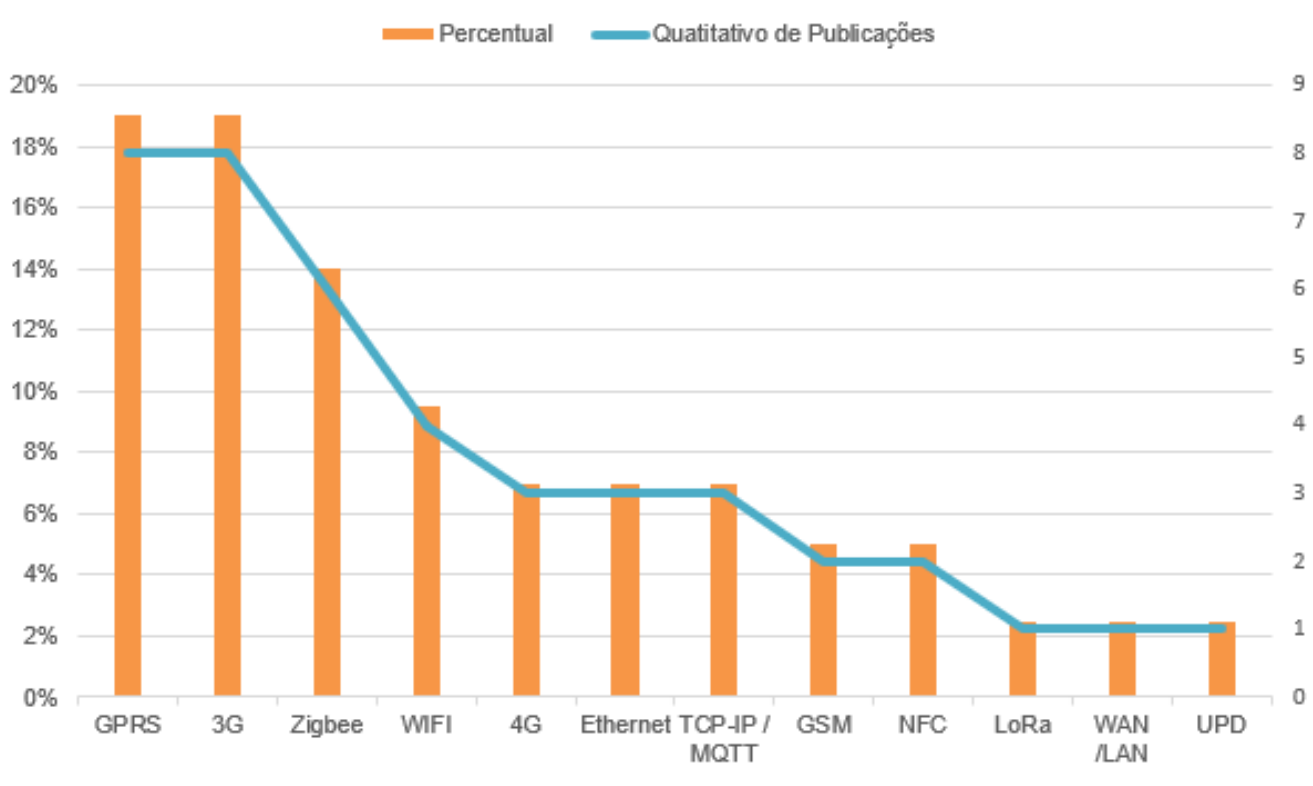

Fonte - Os autores.

O Gráfico 3 e 4 apresentam uma relação importante para com as demais camadas em análise, pois a camada aplicação (interfaces) permite a visualização dos dados coletados pelos sensores, transmitidos por uma rede, vistos em tempo real ou quase em tempo real. Quando comparamos os percentuais das interfaces e as atualizações dos ciclos, percebe-se o uso em $48 \%$ para aplicações com a tecnologia mobile (com uso de smartphones) e $83 \%$ das atualizações dos ciclos vistos pelos usuários realizadas em tempo real. Os principais índices aqui apresentados são justificados pela facilidade de acesso aos aparelhos telefônicos, bem como a ampla cobertura de sinal oferecida pelas operadoras.

Gráfico 3 - Relação Percentual x Quantitativo por camadas loT (aplicação).

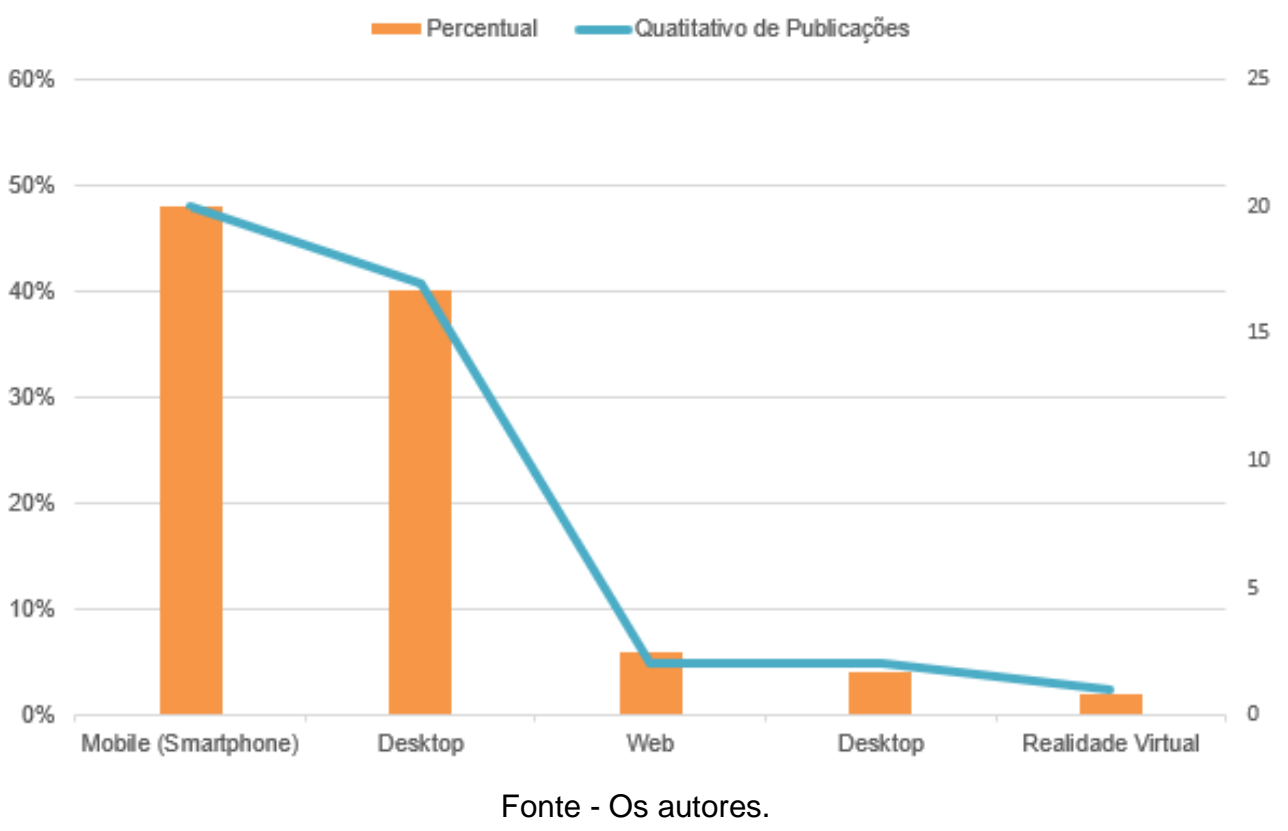


Gráfico 4 - Relação Percentual x Quantitativo por camadas loT (atualização do ciclo).

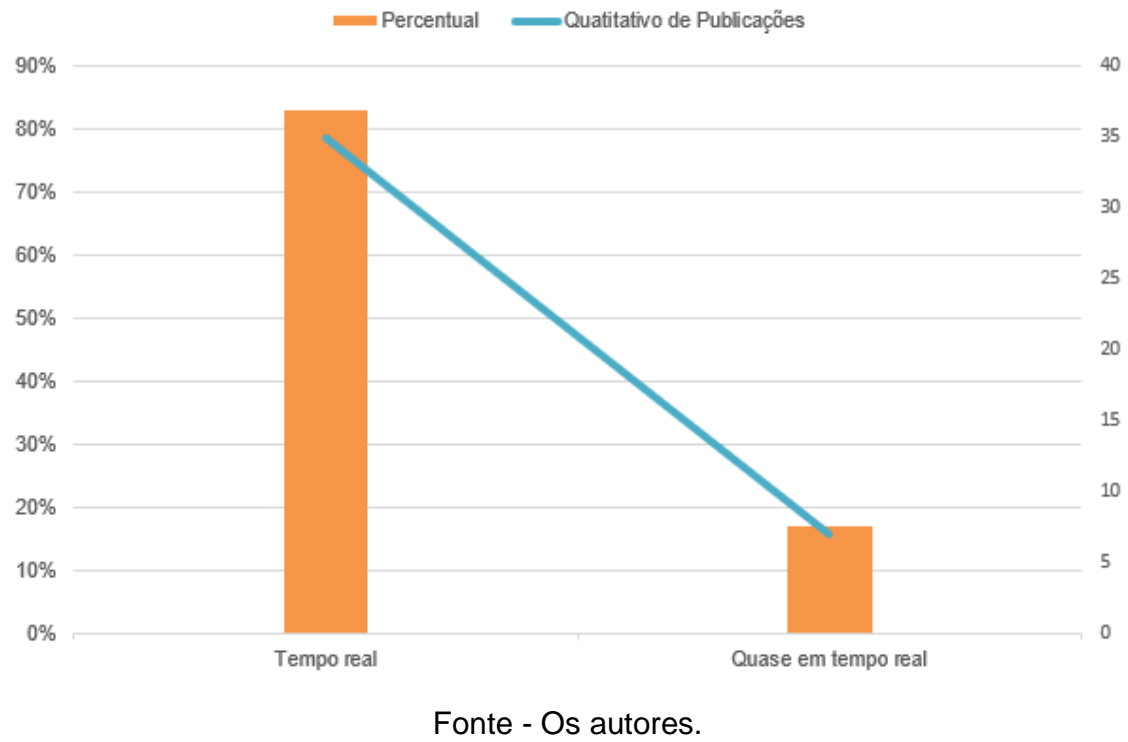

\section{Principais camadas de domínio GIS}

Nos artigos selecionados, as principais funcionalidades GIS aplicam-se a uma utilização de base de dados, ambiente para armazenamento, software para manipulação ou processamentos, plataforma de visualização e formatos de arquivos.

As amostras relacionadas utilizaram em $42 \%$ das pesquisas o Global Navigation Satellite System (GNSS) enquanto referência para as bases de dados geográficos e cerca $31 \%$ referenciaram os trabalhos às imagens de satélites de plataformas open source, como por exemplo: Open Street Map (OSM), Google Maps e Bing (Gráfico 5).

Gráfico 5 - Relação Percentual x Quantitativo por camadas GIS (base de dados).

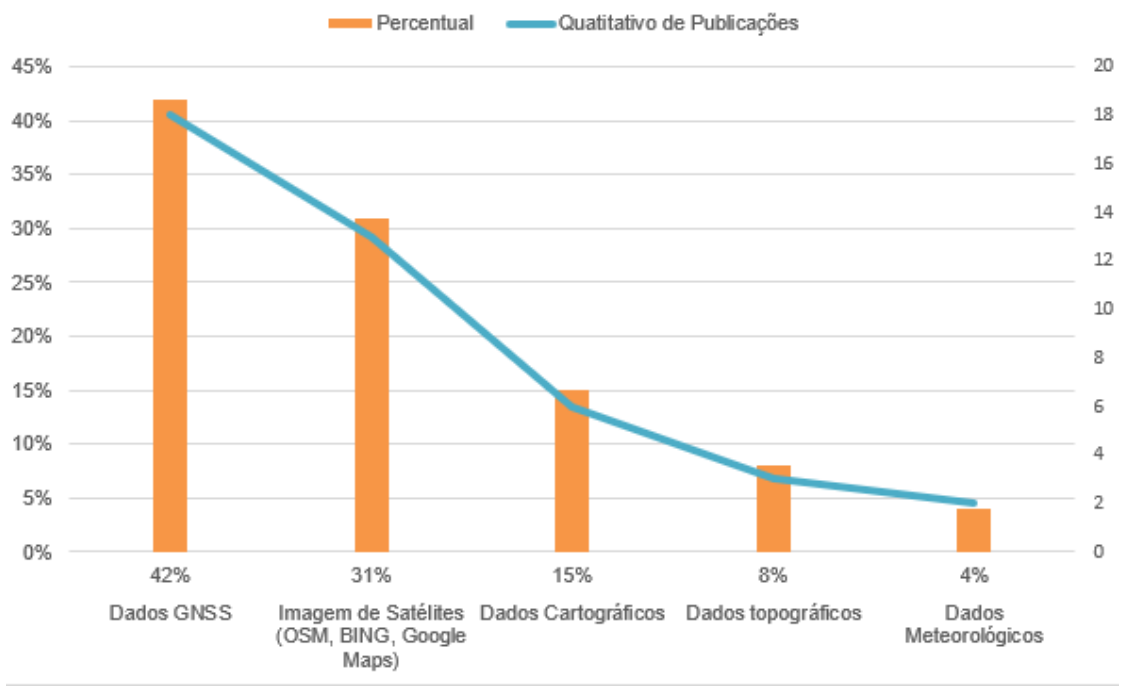

Fonte - Os autores.

No quesito associado aos principais softwares para manipulação e processamentos de dados, ainda é muito comum a utilização dos produtos da empresa americana Environmental Systems Research Institute (ESRI). As análises apontam que cerca de 33\% dos trabalhos utilizaram o ArcGIS como software de referência. O Google Maps com 33\% e o software livre QGIS, aparecem em seguida como principais preferências. 
Gráfico 6 - Relação Percentual x Quantitativo por camadas GIS (softwares).

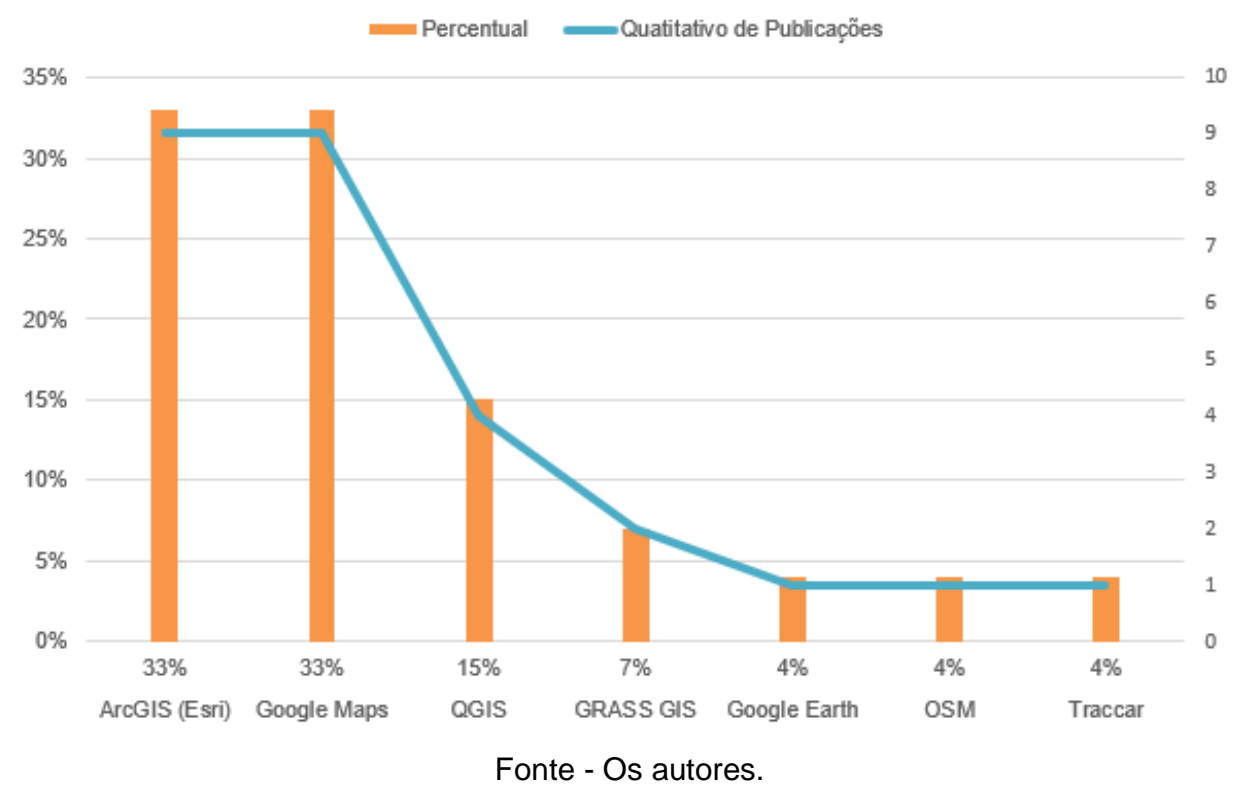

Foote e Kirvan (1997), apontam para o desenvolvimento do sistema WebGIS mediante os esforços para o desenvolvimento de novas funcionalidades GIS implantadas diretamente na internet e em intranets, promovendo assim, uma maior disponibilização das informações geográficas. Como sinalizado outrora, verifica-se em tempos atuais que $58 \%$ das pesquisas utilizadas na RSL desenvolveram sistemas WebGIS para visualização e análise dos dados integrados em ambiente IOT (Gráfico 7).

Gráfico 7 - Relação Percentual x Quantitativo por camadas GIS (ambiente de visualização).

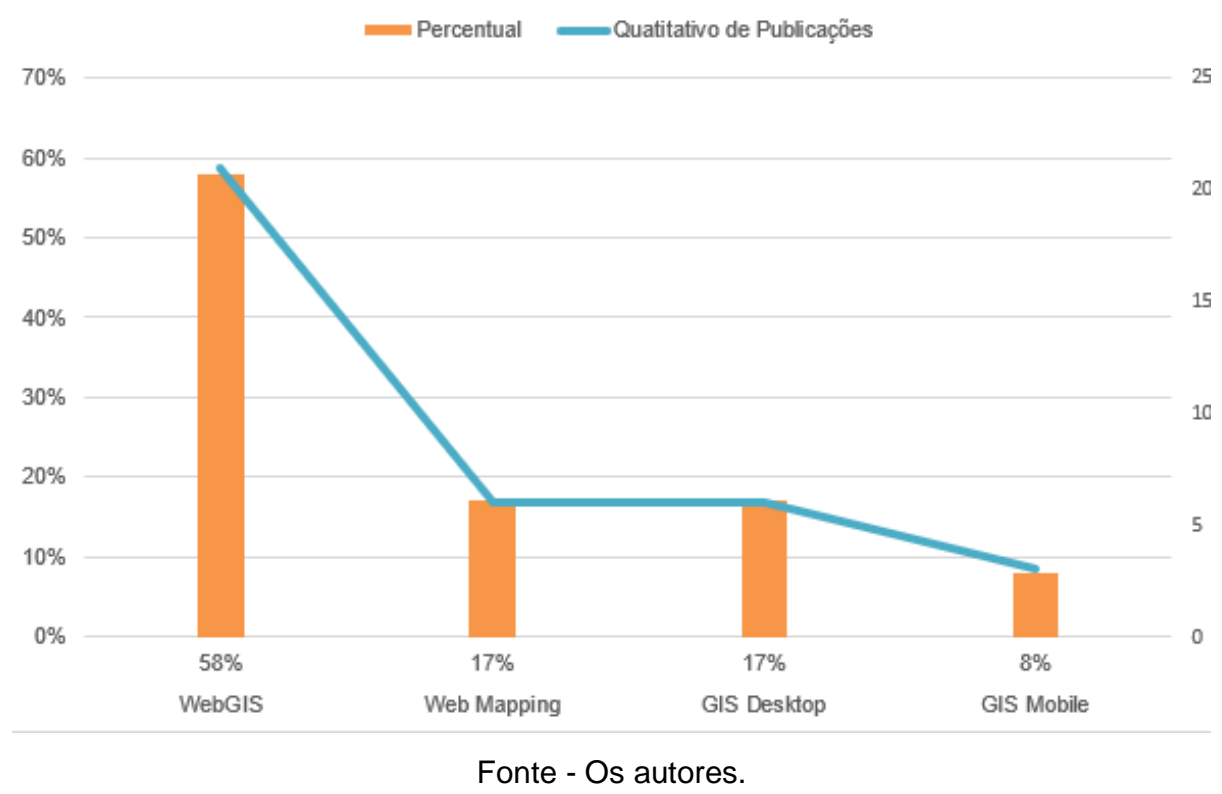

Nos aspectos associados aos métodos de armazenamento, nota-se uma boa variedade de opções para implementação e gerenciamento dos dados espaciais. Todavia, $50 \%$ das pesquisas utilizaram enquanto referência o sistema gerenciador de banco de dados PostgreSQL (Gráfico 8). Acredita-se, que tal aderência seja em consequência deste banco de dados relacional possuir uma extensão para uso de dados geográficos. A extensão denominada PostGIS é potencializada pelo fato de ser um sistema de código aberto.

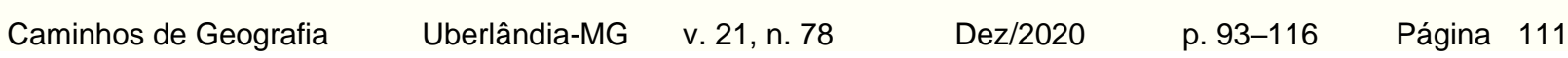


Gráfico 8 - Relação Percentual x Quantitativo por camadas GIS (armazenamento).

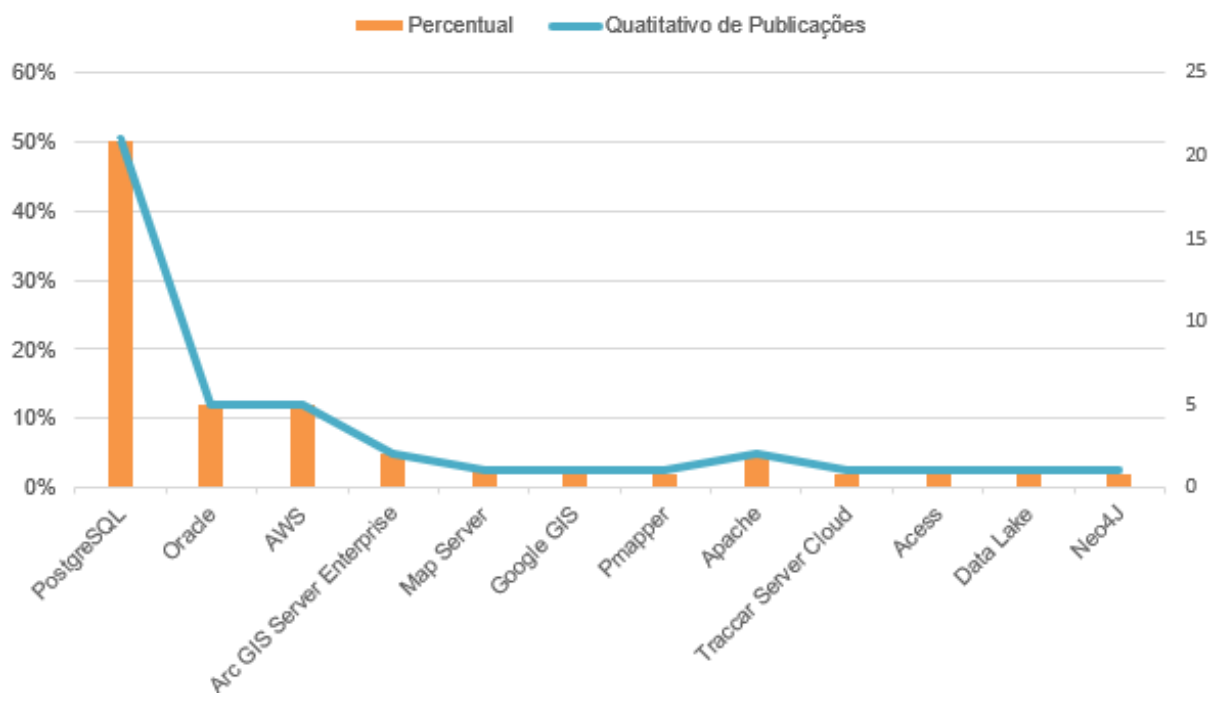

Fonte - Os autores.

Neste levantamento, também foi possível identificar os principais formatos de arquivos, oriundos de sensores ou de modelos geométricos, aos quais, sejam possíveis a sua visualização em ambiente GIS. Para tanto, o formato GeoJSON Format Specification destaca-se em $42 \%$ das pesquisas investigadas. Conforme indicado por GeoJSON (2016), este é um formato para codificar uma variedade de estruturas de dados geográficos na forma de objetos JavaScript Object Notation (JSON).

O GeoJSON é um formato amplamente utilizado para representar geoinformação pela web. Um objeto GeoJSON pode representar uma geometria, um recurso ou uma coleção de recursos suportando para os seguintes tipos de geometria: Point, LineString, Polygon, MultiPoint, MultiLineString, MultiPolygon e GeometryCollection (ISIKDAG; PILOK, 2016). Este formato pode ser originado mediante uma conversão dos dados ainda no padrão Industry Foundation Classes (IFC). De acordo com Gunduz et al. (2017) o GeoJSON pode ser visualizado nas principais ferramentas de manipulação de dados espaciais como por exemplo: ArcGIS, Google Maps e QGIS, ambas acima justificadas. O Gráfico 9 apresenta a distribuição dos demais formatos utilizados.

Gráfico 9 - Relação Percentual x Quantitativo por camadas GIS (formato dos dados).

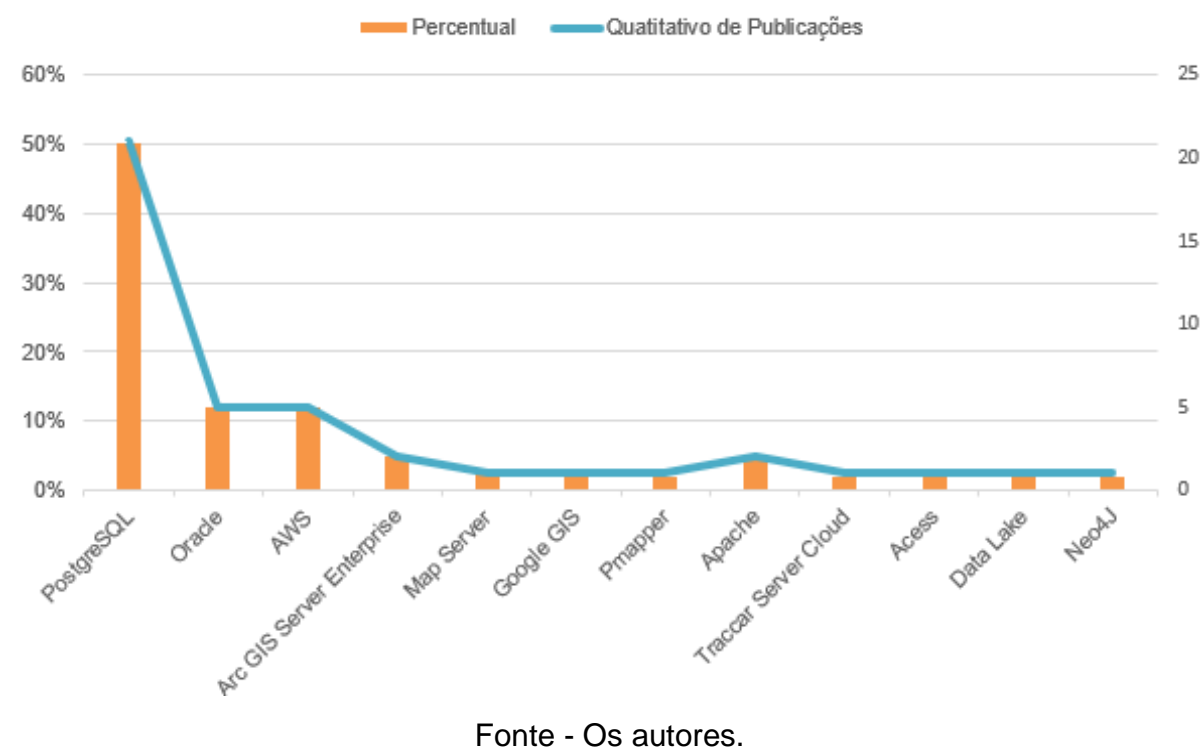




\section{CONSDERAÇÕES FINAIS}

Compreender os paradigmas que abarcam os diferentes métodos, tecnologias e aplicações integradas nos domínios IoT e GIS é algo de suma importância atualmente. A possibilidade de diferentes aplicações nas mais variadas áreas de conhecimento, permitem o desenvolvimento de projetos em prol da melhor qualidade de vida das pessoas.

A Revisão Sistemática de Literatura permitiu explicitar como outros países estão desenvolvendo suas pesquisas nesta área de conhecimento, vislumbrando as tecnologias utilizadas, principais aplicações, identificação de lacunas de pesquisa, bem como as tendências tecnológicas para tal integração.

Este artigo buscou analisar através da estatística descritiva as diferentes abordagens, contribuições e soluções quanto as aplicações relacionadas à integração da loT e GIS. Nesta literatura, apresentamos uma exploração iniciada com 415 trabalhos publicados na base de dados Scopus, Science Direct e Web of Science, entre 2000 e 2018.

A conquista da integração desses dois conceitos e tecnologias terá um impacto significativo na solução dos mais diversos problemas cotidianos. No entanto, como a loT e o GIS foram desenvolvidos originalmente para diferentes propósitos, vários desafios estão sendo encontrados para a integração. Percebe-se a necessidade de maiores esclarecimentos para o desenvolvimento de uma arquitetura solida, que vise principalmente, a utilização de softwares e plataformas open source e de baixo custo.

O presente estudo insere-se em uma pesquisa de doutorado, desenvolvida no Programa de PósGraduação em Engenharia Civil (PPEC) da Universidade Federal da Bahia (UFBA).

\section{AGRADECIMENTOS}

Agradecemos à Coordenação de Aperfeiçoamento de Pessoal de Nível Superior (CAPES), à Universidade Federal da Bahia (UFBA) e ao Karlsruher Institut für Technologie (KIT) pelo apoio dado à pesquisa. Este trabalho foi desenvolvido com apoio do Programa de Cooperação Internacional do CAPES/DAAD - PROBAL, no KIT, na Alemanha, projeto oㅜ 88887.144164/2017-00. Financiado pela CAPES.

\section{REFERENCIAS}

AHAMED, E. et al. The role of big data analytics in internet of things. Computer. Networks. p. 459471, 2017. https://doi.org/10.1016/j.comnet.2017.06.013

AMORIM, A. L. Cidades Inteligentes e City Information Modeling. In: CONGRESS OF THE IBEROAMERICAN SOCIETY OF DIGITAL GRAPHICS. 20., 2016, Buenos Aires. Proceedings [...]. Argentina: 2016. p. 481-488. https://doi.org/10.5151/despro-sigradi2016-440

AN, M. et al. Remote monitoring system of the rivers and lakes' water quality based on GPRS and 3G technology. Advanced Materials Research, v. 898, p. 618-623, 2014. https://doi.org/10.4028/www.scientific.net/AMR.898.618

ATHANASIOU, T. et al. A Web-Geographical Information System for Real Time Monitoring of Arachthos River. IFAC Papers online, v. 51, n. 30, p. 384-389, 2018. https://doi.org/10.1016/j.ifacol.2018.11.335

ATZORI, L.; IERA, A.; MORABITO, G. The internet of things: A survey. Computer Networks, p. 2787-2805, 2010. https://doi.org/10.1016/i.comnet.2010.05.010

BELLO, O.; ZEADALLY, S. Toward efficient smartification of the Internet of Things (IoT) services. Computer System, 2017.

BOTTACCIOLI, L. et al. Building Energy Modelling and Monitoring by Integration of loT Devices and Building Information Models. International Computer Software and Applications, v. 1, p. 914-922, 2017a. https://doi.org/10.1109/COMPSAC.2017.75

BOTTACCIOLI, L. et al. A Novel Integrated Real-time Simulation Platform for Assessing Photovoltaic Penetration Impacts in Smart Grids. Energy Procedia, v. 111, p. 780-789, 2017b. https://doi.org/10.1016/i.egypro.2017.03.240

$\begin{array}{lllll}\text { Caminhos de Geografia } \quad \text { Uberlândia-MG } & \text { v. 21, n. } 78 & \text { Dez/2020 } & \text { p. 93-116 } & \text { Página } 113\end{array}$


BURROUGH, P. A.; MCDONNELL, R.A. Principles of geographical information systems. Oxford: Oxford University Press, 1998.

CADAVID, H. et al. Towards a smart farming platform: From loT-based crop sensing to data analytics. Communications in Computer and Information Science, v. 885, p. 237-251, 2018. https://doi.org/10.1007/978-3-319-98998-3 19

CHEN, Z. et al. Quasi real-time evaluation system for seismic disaster based on internet of things. In: INTERNATIONAL CONFERENCES ON INTERNET OF THINGS AND CYBER, PHYSICAL AND SOCIAL COMPUTING. 2011. Dalian, Proceedings [...]. Dalian. 2011. p. 520-524. https://doi.org/10.1109/iThings/CPSCom.2011.112

CHERRADI, G. et al. Real-Time Microservices Based Environmental Sensors System for Hazmat Transportation Networks Monitoring. Transportation Research, v. 27, p. 873-880, 2017 a. https://doi.org/10.1016/j.trpro.2017.12.087

CHERRADI, $G$ et al. Real-Time HazMat Environmental Information System: A micro-service based architecture. Computer Science, v. 109, p. 982-987, 2017b. https://doi.org/10.1016/i.procs.2017.05.457

DEBAUCHE, O. et al. Irrigation pivot-center connected at low cost for the reduction of crop water requirements. In: INTERNATIONAL CONFERENCE ON ADVANCED COMMUNICATION TECHNOLOGIES AND NETWORKING. 2018. Marrocos. Proceedings [...]. Marrocos: 2018. p. 1-9. https://doi.org/10.1109/COMMNET.2018.8360259

DRAZ, U. et al. A real-time smart dumpsters monitoring and garbage collection system. In: INTERNATIONAL CONFERENCE ON AEROSPACE SCIENCE AND ENGINEERING. 2017. Islamabad, Proceedings [...]. Paquistão: 2017. p. 1-8. https://doi.org/10.1109/ICASE.2017.8374268

FANG, S. et al. An integrated system for regional environmental monitoring and management based on internet of things. Transactions on Industrial Informatics, v. 10, n. 2, p. 1596-1605, 2014. https://doi.org/10.1109/TII.2014.2302638

FOOTE, K. E.; KIRVAN, A. P. WebGIS, NCGIA Core Curriculum. In: GIScience Available, 1997. Disponível em: http://www.ncgia.ucsb.edu/ giscc/units/ u133/u133.html. Acesso em: 13 jan. 2019.

FORTINO, G. et al. Modeling opportunistic loT services in open loT ecosystems, In: WORKSHOP FROM OBJECTS TO AGENTS, 2017. Bologna Proceedings [...]. Itália: 2017. p. 90-95.

GAN, Q. Research on multi-dimensional logistics based on the internet of things. Open Automation and Control Systems Journal, v. 7, n. 1, p. 2051-2056, 2015. https://doi.org/10.2174/1874444301507012051

GAO, D; ZHANG, Y; LI, X. The internet of things for electric vehicles: Wide area charging-swap information perception, transmission and application. Advanced Materials Research. v. 608-609, p. 1560-1565, 2013. https://doi.org/10.4028/www.scientific.net/AMR.608-609.1560

GUNDUZ, M.; ISIKDAG, U.; BASARANER, M. Integration of BIM, Web Maps and loT for supporting comfort analysis. ISPRS, 2017, Turquia. Proceedings [...]. Turquia: 2017. p. 221-227. https://doi.org/10.5194/isprs-annals-IV-4-W4-221-2017

GUBBI, J. et al. Internet of Things (IoT): a vision, architectural elements, and future directions, Computer System. p. 1645-1660, 2013. https://doi.org/10.1016/j.future.2013.01.010

GUTIERREZ, J. M. et al. Smart Waste Collection System Based on Location Intelligence. Procedia Computer Science, v. 61, p. 120-127, 2015. https://doi.org/10.1016/..procs.2015.09.170

HAMIDI, S. R. et al. Industry 4.0 urban mobility: goNpark smart parking tracking module. ACM INTERNATIONAL CONFERENCE, Tóquio. Proceedings [...]. Tóquio: 2017. p. 503-507.JAGWANI, Priti. https://doi.org/10.1145/3162957.3163042

KUMAR, Manoj. IoT powered vehicle tracking system (VTS). Computer Science, v. 10963 LNCS: p. 488-498, 2018. https://doi.org/10.1007/978-3-319-95171-3 38

HUANG, J. et al. 3D WebGIS-based platform for debris flow early warning: A case study. Engineering Geology, v. 197, p. 57-66, 2015. https://doi.org/10.1016/i.enggeo.2015.08.013

$\begin{array}{lllll}\text { Caminhos de Geografia } \quad \text { Uberlândia-MG } & \text { v. 21, n. 78 } \quad \text { Dez/2020 } & \text { p. 93-116 Página } 114\end{array}$


HUANG, X; ZHU, Wei; LU, Dongxin. Underground miners localization system based on ZigBee and WebGIS. In: INTERNATIONAL CONFERENCE ON GEOINFORMATICS, 18., 2010. Beijing. Proceedings [...]. China: 2010. p. 1-5. https://doi.org/10.1109/GEOINFORMATICS.2010.5567542

ISIKDAG, U.; PILOUK, M. Integration of GEO-sensor feeds and event consumer services for real-time representation of IOT nodes. In: INTERNATIONAL ARCHIVES OF THE PHOTOGRAMMETRY, REMOTE SENSING AND SPATIAL INFORMATION SCIENCES, 2016. Praga. Proceedings [...]. República Checa: 2016. p. 267-274. https://doi.org/10.5194/isprs-archives-XLI-B4-267-2016

ISIKDAG, U. BIM and IoT: A synopsis from GIS perspective. ISPRS, 2015, Malásia. Proceedings [...]. Malásia: 2015. p. 33-38. https://doi.org/10.5194/isprsarchives-XL-2-W4-33-2015

JAGWANI, P.; KUMAR, M. IoT powered vehicle tracking system (VTS). Computer Science, v. 10963 LNCS: p. 488-498, 2018. https://doi.org/10.1007/978-3-319-95171-3 38

JIA, B.; YANG, Y.; CUI, C. The construction of security supervision platform for fireworks and firecrackers based on IOT. Applied Mechanics and Materials. v. 191, p. 195-198, 2012. https://doi.org/10.4028/www.scientific.net/AMM.190-191.195

JIN, J. et al. An Information Framework for Creating a Smart City through Internet of Things. Internet of Things Journal, v. 1, p. 112-121, 2014. https://doi.org/10.1109/JIOT.2013.2296516

KIAVARZ, H. et al. BIM-GIS oriented intelligent knowledge discovery. International Archives of the Photogrammetry, Remote Sensing and Spatial Information Sciences - ISPRS Archives, p. 79-82, 2018. https://doi.org/10.5194/isprs-archives-XLII-4-W10-79-2018

KOTIKOV, J. GIS-modeling of multimodal complex road network and its traffic organization.

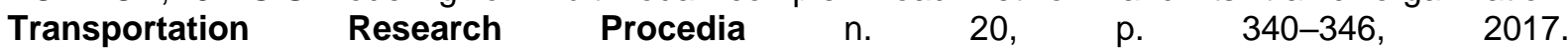
https://doi.org/10.1016/i.trpro.2017.01.043

LI, D. et al. An RFID and sensor web-enabled smart electric power equipment inspection system. $\begin{array}{lllll}\text { International Conference on } & \text { Geoinformatics, } & \text { v. } & 17, & \end{array}$ https://doi.org/10.1109/GEOINFORMATICS.2017.8090921

LIU, C.; MENG, X.; FAN, Y. Determination of routing velocity with GPS floating car data and webGISbased instantaneous traffic information dissemination. Journal of Navigation, v. 61, n. 2, p. 337-353, 2008. https://doi.org/10.1017/S0373463307004547

LUCHETTI, G. et al. Whistland: An augmented reality crowd-mapping system for civil protection and emergency management. ISPRS, 2017, Brecce. Proceedings [...]. Brecce: 2017. p. 1-18. https://doi.org/10.3390/ijgi6020041

MANGIAMELI, M.; MUSSUMECI, G. Real time integration of field data Into a GIS platform for the management of hydrological emergencies. ISPRS Archives, v. 40, n. 5W3, p. 153-158, 2013. https://doi.org/10.5194/isprsarchives-XL-5-W3-153-2013

MANVILLE, C. et al. Smart cities in the Europe, 2014.

MAREK, L. et al. Real-time environmental sensors to improve health in the sensing city. ISPRS Archives, p. 729-733, 2016. https://doi.org/10.5194/isprsarchives-XLI-B2-729-2016

MO, X; QIU, X. J.; SHEN, S. An loT-based system for water resources monitoring and management. In: INTERNATIONAL CONFERENCE ON INTELLIGENT HUMAM-MACHINE SYSTEMS AND CYBERNETICS, 2., 2015, Zhejian. Proceedings [...]. Zhejian: 2015. p. 365-368.

NOURJOU, R.; HASHEMIPOUR, M. Smart Energy Utilities based on Real-Time GIS Web Services and Internet of Things. Computer Science, v. 110, p. 8-15, 2017. https://doi.org/10.1016/i.procs.2017.06.070

PASQUALE, C. et al. Virtual data warehouse architecture for real-time WebGIS. In: INTERNATIONAL CONFERENCE ON VIRTUAL ENVIRONMENTS, HUMAN-COMPUTER INTERFACES AND MEASUREMENT SYSTMS., 2008, Instambul. Proceedings [...]. Turquia: 2008. p. 80-85. https://doi.org/10.1109/VECIMS.2008.4592757

PENG, S. et al. Design of an loT-BIM-GIS Based Risk Management System for Hospital Basic Operation. INTERNATIONAL SYMPOSIUM ON SERVICE ORIENTED SYSTEM, 11., 2017, San Francisco. Proceedings [...]. San Francisco: 2017. p. 69-74. https://doi.org/10.1109/SOSE.2017.22 
PIROTTI, F.; GUARNIERI, A.; VETTORE, A. Collaborative Web-GIS Design: A Case Study for Road Risk Analysis and Monitoring. Transactions in GIS, v. 15, n. 2, p. 213-226, 2011. https://doi.org/10.1111/j.1467-9671.2011.01248.x

SERRANO, S. et al. GIS design application for "Sierra Morena Honey" designation of origin. Computers and Electronics in Agriculture. p. 307-317, 2008. https://doi.org/10.1016/i.compag.2008.07.001

SCIORTINO, R. et al. A webGIS-based system for real time shelf life prediction. Computers and Electronics in Agriculture, v. 127, p. 451-459, 2016. https://doi.org/10.1016/j.compag.2016.07.004

SOTO-GARCIA, $M$. et al. The role of information and communication technologies in the modernization of water user associations' management. Computers and Electronics in Agriculture, v. 98 , p. 121-130, 2013. https://doi.org/10.1016/i.compag.2013.08.005

TAN, T. H. et al. Development of an emergency medical service system based on wireless networks and real-time traffic information. In: INTERNATIONAL CONFERENCE ON COMPUTERIZED HEALTHCARE. 2012. Hong Kong. Proceedings [...]. China: 2012. p. 35-42. https://doi.org/10.1109//CCH.2012.6724467

TANG, C. P. et al. WSID identification platform of heterogeneous networks based on RFID and WSN. In: INTERNATIONAL CONFERENCE ON RFID-TECHNOLOGY AND PLICATIONS. 2010, Guangzhou. Proceedings [...]. Guangzhou. 2010. p. 217-221. https://doi.org/10.1109/RFIDTA.2010.5529935

THANOS, G. K. et al. SYNAISTHISI: An loT-powered smart visitor management and cognitive recommendations system. International Society for Optical Engineering, v. 9842, p. 1-18, 2016. https://doi.org/10.1117/12.2224045

TRILLIES, S. et al. Deployment of an open sensorized platform in a smart city context. Future Generation Computer Systems, v. 76, p. 221-233. 2017. https://doi.org/10.1016/i.future.2016.11.005

WALRAVENS, N.; BALLON, P. Platform business models for smart cities: from control and value to governance and public value, Communications Magazine. v. 51, n. 6, p. 72-79, 2013. https://doi.org/10.1109/MCOM.2013.6525598

WU, C. et al. A NoSQL-SQL hybrid organization and management approach for real-time geospatial data: A case study of public security video surveillance. International Journal of Geo-Information, v. 6, n. 1, 2017. https://doi.org/10.3390/ijgi6010021

YUAN, L; ZHAO, J. Construction of the system framework of Spatial Data Warehouse in Internet of Things environments. INTERNATIONAL CONFERENCE ON ADVANCED COMPUTATIONAL INTELLIGENCE. 5., China. Proceedings [...]. China: $2012 . \quad$ p. 888-892. https://doi.org/10.1109/ICACI.2012.6463121

YUAN, H.; ZHONG, H.; LAI, D. One environmental monitoring early-warning system research by RIA technology. Applied Mechanics and Materials. v. 295-298, p. 888-892, 2013. https://doi.org/10.4028/www.scientific.net/AMM.295-298.888

Recebido em: 06/03/2020

Aceito para publicação em: 11/08/2020 